\title{
UNITARY REPRESENTATIONS OF DIAMOND GROUPS
}

\author{
DETLEV POGUNTKE
}

\section{Dedicated to Professor Horst Herrlich on His Sixtieth Birthday}

Recently Ludwig, [14], has considered the topology on the unitary dual of so-called diamond groups. Those are simply connected solvable Lie groups which can be written as semidirect products $S \ltimes N$, where $N$ is the Heisenberg group in a certain dimension, $S$ is abelian, i.e. isomorphic to some $\mathrm{R}^{d}, S$ fixes the center of $N$, and $S$ acts on the Lie algebra $\mathfrak{n}$ of $N$ by semisimple linear transformations with spectra in the unit circle. In other words, the action is given by a continuous homomorphism $\alpha$ from $S$ into an anisotropic torus of the appropriate symplectic group. These groups are of particular interest because in some sense, compare [14], they are the minimal solvable Lie groups where the infinite-dimensional irreducible unitary representations (or rather the primitive ideals of infinite codimension in the non type I case) cannot be obtained by inducing from proper subgroups. Nevertheless, using holomorphic induction the set Priv $C^{*}(S \ltimes N)$ of primitive ideals in the $C^{*}$ algebra of $S \ltimes N$ can be parametrized (as can be done for all simply connected solvable Lie groups, see [19]). In particular, one obtains a bijective map from the space of $S \ltimes N$-quasi-orbits in the linear dual $(\mathfrak{s} \ltimes \mathfrak{n})^{*}$ of the Lie algebra of $S \ltimes N$ onto a certain quotient of Priv $C^{*}(S \ltimes N)$, which may be considered as a variant of Kirillov's orbit picture. The main result of [14] tells that this bijection is open; the continuity was not investigated.

Both Ludwig's and the present article may be regarded as part of the program to determine the topology on the dual space of a connected solvable Lie group which still is an open question. Previous experiences put in evidence that the continuity of the map from the parameter space into the dual is easier to establish than its openness: Kirillov, [12], proved the continuity of his map in the case of nilpotent Lie groups in 1962, while only 1973 Brown showed its openness, [4]. The continuity in the case of exponential groups was established by Pukanszky, [18], in 1968, the openness only in 1994 by Leptin and Ludwig, [13]. Similarily, the continuity of the Dixmier

Received February 17, 1997. 
map for universal enveloping algebras of solvable Lie algebras was shown in 1970 by Berline and Duflo, [5], while it took until 1991 to obtain its openness, Mathieu, [15].

In this paper we study more general "diamond groups" $S \ltimes N$ by assuming only that $S$ is a second countable locally compact abelian group acting on the Heisenberg group $N$ as above. Even more general we consider covariance algebras $C^{*}\left(S, C^{*}(N) \otimes \mathscr{B}, T\right)$ where $\mathscr{B}$ is a commutative $C^{*}$ algebra, and $S$ acts diagonally on $C^{*}(N) \otimes \mathscr{B}$, on $C^{*}(N)$ as before and arbitrarily on $\mathscr{B}$. For such algebras we determine the topological space Priv $C^{*}\left(S, C^{*}(N) \otimes\right.$ $\mathscr{B}, T)$. As consequences we get another proof of Ludwig's result for the original case and furthermore that the above mentioned bijection is in general not continuous.

The case $\mathscr{B}=\mathrm{C}$ and $S=$ maximal anisotropic torus in the appropriate symplectic group plays a crucial role in our approach. In this case, which is treated in the second section, we determine the dual topology by an explicit computation of matrix coefficients. The case of more general diamond groups is reduced to this particular one by means of Takai duality. In this reduction commutative (group) $C^{*}$-algebras $\mathscr{B}$ enter the scene naturally mainly for that reason we study the general setting described in the last paragraph. This material forms the content of the third section, which is the main body of this article.

In the fourth section we return to groups and discuss some special cases. One of the examples leads to the disappointing conclusion that a natural guess for the topology on the set of the Pukanszky parameters indeed makes the Pukanszky-Kirillov map from the parameter set into the dual space discontinuous. The article is finished by posing a problem on the duals of a class of relatively concrete $C^{*}$-algebras, which in my opinion should be solved before turning to general solvable Lie groups.

\section{$\S$ 1. Notations, Coadjoint Orbits, Metabelian Groups}

In this first section we fix some notations for the whole article and provide some information on coadjoint orbits for diamond groups.

Let $\mathfrak{n}$ be the Heisenberg algebra in dimension $2 n+1$. In this article we shall work with the "complex" picture, i.e., $\mathfrak{n}$ is the real vector space $\mathrm{C}^{n} \times \mathrm{R}$ with bracket

$$
\left[(z, t),\left(z^{\prime}, t^{\prime}\right)\right]=\left(0,-\operatorname{Im}\left(z \overline{z^{\prime}}\right)\right)
$$

where $z \overline{z^{\prime}}$ abbreviates $\sum_{j=1}^{n} z_{j} \overline{z_{j}^{\prime}}$ if $z=\left(z_{1}, \ldots, z_{n}\right), z^{\prime}=\left(z_{1}^{\prime}, \ldots, z_{n}^{\prime}\right)$. Accordingly the group multiplication on $N=\mathrm{C}^{n} \times \mathrm{R}$ is given by

$$
(z, t)\left(z^{\prime}, t^{\prime}\right)=\left(z+z^{\prime}, t+t^{\prime}-\frac{1}{2} \operatorname{Im}\left(z \overline{z^{\prime}}\right) .\right.
$$


Let $U$ be an $n$-dimensional torus with universal covering exp $: \mathfrak{u} \rightarrow U$. We fix an identification $\zeta$ of $U$ with a certain subgroup of $\operatorname{Aut}(N)$ (or of $\operatorname{Aut}(\mathfrak{n}))$, namely

$$
\zeta(u)\left(z_{1}, \ldots, z_{n}, t\right)=\left(\zeta_{1}(u) z_{1}, \ldots, \zeta_{n}(u) z_{n}, t\right)
$$

for a set $\zeta_{1}, \ldots, \zeta_{n}$ of free generators of the Pontrjagin dual $U^{\wedge}$.

Definition 1.4. A (generalized) diamond group is a semidirect product $S \ltimes N$ where $S$ is a second countable locally compact abelian group, and the action of $S$ on $N$ is given by a continuous homomorphism $\alpha: S \rightarrow U$, i.e., the multiplication in $S \ltimes N$ is given by

$$
(s, x)\left(s^{\prime}, x^{\prime}\right)=\left(s s^{\prime}, \zeta\left(\alpha\left(s^{\prime}\right)^{-1}\right)(x) x^{\prime}\right) .
$$

Very often we shall drop $\zeta$ and write

$$
(s, x)\left(s^{\prime}, x^{\prime}\right)=\left(s s^{\prime}, \alpha\left(s^{\prime}\right)^{-1}(x) x^{\prime}\right)
$$

by a slight abuse of notation or, more explicitly,

$$
\begin{aligned}
& (s, z, t)\left(s^{\prime}, z^{\prime}, t^{\prime}\right)= \\
& \left(s s^{\prime}, \alpha_{1}\left(s^{\prime}\right)^{-1} z_{1}+z_{1}^{\prime}, \ldots, \alpha_{n}\left(s^{\prime}\right)^{-1} z_{n}+z_{n}^{\prime}, t+t^{\prime}-\frac{1}{2} \sum_{j=1}^{n} \operatorname{Im}\left(\alpha_{j}\left(s^{\prime}\right)^{-1} z_{j} \overline{z_{j}^{\prime}}\right)\right)
\end{aligned}
$$

with $\alpha_{1}, \ldots, \alpha_{n} \in S^{\wedge}$. If $S=U$ and $\alpha=$ Id the group $U \ltimes N$ will be called the full diamond group and will be denoted by $G$ throughout this article.

If $S$ is a connected Lie group, i.e., $S$ is isomorphic to $\mathrm{R}^{a} \times \mathrm{T}^{b}$, with universal covering exp : $\mathfrak{s} \rightarrow S$ we may form the Lie algebra $\mathfrak{s} \ltimes \mathfrak{n}$ of $S \ltimes N$, the bracket being given by

$$
\begin{aligned}
& {\left[(A, z, t),\left(A^{\prime}, z^{\prime}, t^{\prime}\right)\right]=} \\
& \left(0, i d \alpha_{1}(A) z_{1}^{\prime}-i d \alpha_{1}\left(A^{\prime}\right) z_{1}, \ldots, i d \alpha_{n}(A) z_{n}^{\prime}-i d \alpha_{n}\left(A^{\prime}\right) z_{n},-\operatorname{Im}\left(z \overline{z^{\prime}}\right)\right)
\end{aligned}
$$

for $A, A^{\prime} \in \mathfrak{s}$ and $(z, t),\left(z^{\prime}, t^{\prime}\right) \in \mathfrak{n}$ where $d \alpha_{1}, \ldots, d \alpha_{n}$ in the linear dual $\mathfrak{s}^{*}$ are determined by

$$
\alpha_{j}(\exp A)=e^{i d \alpha_{j}(A)}, A \in \mathfrak{s} .
$$

Accordingly for the Lie algebra $\mathfrak{g}=\mathfrak{u} \ltimes \mathfrak{n}$ of the full diamond group $G=U \ltimes N$ we have a similar expression for the bracket, the $d \alpha_{j}$ being replaced by the functionals $d \zeta_{j} \in \mathfrak{u}^{*}$ in the evident meaning.

It is not hard to verify that the exponential map exp : $\mathfrak{1} \ltimes \mathfrak{n} \rightarrow S \ltimes N$ is given by 


$$
\begin{aligned}
& \exp (A, z, t)=\left(\exp A, i z_{1} \frac{e^{-i d \alpha_{1}(A)}-1}{d \alpha_{1}(A)}, \ldots, i z_{n} \frac{e^{-i d \alpha_{n}(A)}-1}{d \alpha_{n}(A)},\right. \\
& \left.t-\frac{1}{2} \sum_{j=1}^{n}\left|z_{j}\right|^{2} \frac{\sin d \alpha_{j}(A)-d \alpha_{j}(A)}{d \alpha_{j}(A)^{2}}\right)
\end{aligned}
$$

for $A \in \mathfrak{s}$ and $(z, t) \in \mathfrak{n}$.

For the adjoint representation $\mathrm{Ad}: S \ltimes N \rightarrow$ Aut $(\mathfrak{s} \ltimes \mathfrak{n})$ at $\left(s_{0}, z_{0}, t_{0}\right)=$ $\left(s_{0}, z_{0}^{(1)}, \ldots, z_{0}^{(n)}, t_{0}\right) \in S \ltimes N$ one finds

$$
\operatorname{Ad}\left(s_{0}, z_{0}, t_{0}\right)(A, z, t)=\left(A, z^{\prime}, t^{\prime},\right) \text { for } A \in \mathfrak{s}, \quad(z, t) \in \mathfrak{n}
$$

where

$$
t^{\prime}=t-\operatorname{Im} z_{o} \bar{z}-\frac{1}{2} \sum_{j=1}^{n} d \alpha_{j}(A)\left|z_{o}^{(j)}\right|^{2}
$$

and $z^{(j)}=\alpha_{j}\left(s_{0}\right)\left(z^{(j)}-i d \alpha_{j}(A) z_{0}^{(j)}\right)$ for $1 \leq j \leq n$ if $z=\left(z^{(1)}, \ldots, z^{(n)}\right)$.

In the sequel we shall very often be concerned with deciding if a given continuous surjective map is also open. This will be done by means of the following well-known lemma.

Lemma 1.9. For topological spaces $X$ and $Y$ satisfying the first axiom of countability, and a continuous surjective map $p: X \rightarrow Y$ the following are equivalent:

(i) $p$ is open.

(ii) For each sequence $\left(y_{k}\right)$ in $Y$ converging to $y$ and each $x \in X$ with $p(x)=y$ there exists a sequence $\left(x_{k}\right)$ in $X$ such that $p\left(x_{k}\right)=y_{k}$ and $\left(x_{k}\right)$ converges to $x$.

(iii) For each sequence $\left(y_{k}\right)$ in $Y$ converging to $y$ there exists a subsequence $\left(y_{k_{\ell}}\right)$, an $x \in X$ with $p(x)=y$, and a sequence $\left(x_{\ell}\right)$ in $X$ such that $p\left(x_{\ell}\right)=y_{k_{\ell}}$, and $x_{\ell}$ converges to $x$.

Under these circumstances the equivalence relation $\sim$ on $X$ defined by $p$, i.e., $x \sim x^{\prime}$ if $p(x)=p\left(x^{\prime}\right)$, is open, and $Y$ is homeomorphic to the quotient space $X / \sim$.

Before turning to coadjoint orbits for diamond groups we consider semidirect products of connected abelian Lie groups, more general than those appearing as quotients of diamond groups modulo the center $Z N$ of $N$.

Definition 1.10. Let $Q$ be a connected Lie group with Lie algebra $\mathfrak{q}$. A real linear functional $f$ on $Q$ is called integrable if there exists a (unique) unitary character $\chi_{f}$ on the connected component $\left(Q_{f}\right)_{0}$ of the stabilizer of $f$ in $Q$ (w.r.t. the coadjoint action) such that $\chi_{f}(\exp X)=e^{i f(X)}$ for $X$ in $\mathfrak{q}_{f}=$ 
Lie algebra of $Q_{f}$. The collection of all integrable functionals is denoted by $\mathfrak{q}_{\text {int }}$.

Suppose now in addition that $Q$ is a semidirect product $S \ltimes M$ of abelian groups $S$ and $M$ where $M$ is simply connected, i.e., $S$ is isomorphic to $\mathrm{R}^{a} \times \mathrm{T}^{b}$ and $M$ is isomorpic to $\mathrm{R}^{d}$ for some $a, b, d$. An $f \in \mathfrak{q}_{\text {int }}^{*}$ is called aligned if $f\left(\operatorname{ker}\left(\left.\exp \right|_{\mathfrak{s}}\right)\right) \subset 2 \pi \mathrm{Z}, \mathfrak{s}=$ Lie $(S)$, i.e., there is a unitary character $\sigma_{f}$ on $S$ with differential $\left.f\right|_{\mathfrak{s}}$

With each $Q=S \ltimes M$ as above we associate several topological spaces. First we introduce two equivalence relations $\sim$ and $\sim_{M}$ on the topological product $S^{\wedge} \times M^{\wedge}$ of the Pontrjagin duals by

$$
(\sigma, \mu) \sim\left(\sigma^{\prime}, \mu^{\prime}\right)
$$

if the closures of $S \mu$ and $S \mu^{\prime}$ coincide (clearly $s \in S$ acts on $\mu \in M^{\wedge}$ by $\left.(s \mu)(x)=\mu\left(s^{-1} x s\right)\right)$ and if $\sigma=\sigma^{\prime}$ on the stabilizer $S_{\mu}\left(=S_{\mu^{\prime}}\right)$, and by

$$
(\sigma, \mu) \sim_{M}\left(\sigma^{\prime}, \mu^{\prime}\right)
$$

if $\mu=\mu^{\prime}$ and $\sigma=\sigma^{\prime}$ on $S_{\mu}$; both relations are open equivalence relations, compare [17].

Secondly we consider the set $\mathfrak{X}$ of all pairs $(f, \vartheta)$, where $f \in \mathfrak{q}_{\text {int }}^{*}$ and $\vartheta \in Q_{f}^{\wedge}$ is an extension of the above character $\chi_{f}$ on $\left(Q_{f}\right)_{0}$. The notion of alignment is extended to $\mathfrak{X}$ :

(1.13) An element $(f, \vartheta) \in \mathfrak{X}$ is called aligned if $f$ is aligned and if the above character $\sigma_{f}$ on $S$ satisfies $\left.\sigma_{f}\right|_{S_{f}}=\left.\vartheta\right|_{S_{f}}$.

We introduce a topology on $\mathfrak{X}$, not Hausdorff in general, as follows, compare [17, 19]. For each open subset $B$ of the vector space $\mathfrak{q}^{*}$ and each finite collection $\mathfrak{A}$ of pairs $(K, V)$ where $K$ is a compact subset of $Q$ and $V$ is an open subset of the torus T let $W(B, \mathfrak{A})$ be the set of all pairs $(f, \vartheta)$ in $\mathfrak{X}$ such that $f \in B$ and $\vartheta\left(K \cap Q_{f}\right) \subset V$ for all $(K, V) \in \mathfrak{A}$. Those sets $W(B, \mathfrak{A})$ form the basis of a topology on $\mathfrak{X}$. Arguing as in [17, Theorem 2.5] one easily sees:

(1.14) A sequence $\left(f_{k}, \vartheta_{k}\right)$ in $\mathfrak{X}$ converges to $(f, \vartheta)$ if and only if $\left(f_{k}\right)$ converges to $f$ in $\mathfrak{q}^{*}$ and if for each subsequence $\left(f_{k_{\ell}}, \vartheta_{k_{\ell}}\right)$ and each convergent sequence $\left(q_{\ell}\right)$ in $Q$ with $q_{\ell} \in Q_{f_{k_{\ell}}}$ one has

$$
\vartheta\left(\lim _{\ell \rightarrow \infty} q_{\ell}\right)=\lim _{\ell \rightarrow \infty} \vartheta_{k_{\ell}}\left(q_{\ell}\right) .
$$

It is routine to check that $Q$ acts continuously on $\mathfrak{X}$ by $q(f, \vartheta)=\left(f^{\prime}, \vartheta^{\prime}\right)$ where $f^{\prime}=\operatorname{Ad}^{*}(q) f$ and $\vartheta^{\prime}(x)=\vartheta\left(q^{-1} x q\right)$ for $x \in Q_{f^{\prime}}=q Q_{f} q^{-1}$.

Our next lemma says among others that there is a canonical homeomorphism from the quotient space $\left(S^{\wedge} \times M^{\wedge}\right) / \sim$ onto the space $\mathfrak{X} / Q$ of $Q$ quasi-orbits in $\mathfrak{X}$. 
Lemma 1.15. Let $Q=S \ltimes M, \mathfrak{X}, \sim$ and $\sim_{M}$ as above.

(i) For any $f \in \mathfrak{q}^{*}$ one has $Q_{f}=S_{f} \ltimes M_{f}, Q_{g}=S_{g} \ltimes M$, and $S_{f}=S_{g}$ where $g:=\left.f\right|_{\mathfrak{m}}, \mathfrak{m}=$ Lie algebra of $M$; the stabilizer $M_{f}$ is connected. Moreover, $S_{q f}=S_{f}$ for all $q \in Q$ where $q f=\operatorname{Ad}^{*}(q)(f)$. This does not mean that $S_{f}$ is normal because $S_{q f}=S \cap Q_{q f}=S \cap q Q_{f} q^{-1}$ is in general different from $q S_{f} q^{-1}$. But all stabilizer groups $Q_{f}$ are invariant under conjugation with elements in $M$.

(ii) For all $f \in \mathfrak{q}^{*}$ one has $M f=Q_{g} f=f+\left(\mathfrak{q} /\left(\mathfrak{s}_{f}+\mathfrak{m}\right)\right)^{*}$ where $g=\left.f\right|_{\mathfrak{m}}$ and $\mathfrak{s}_{f}=$ Lie $\left(S_{f}\right)=\{A \in \mathfrak{s} \mid f([A, \mathfrak{q}])=0\}$.

(iii) For each $f \in \mathfrak{q}_{\text {int }}^{*}$ the character $\chi_{f}$ on $\left(Q_{f}\right)_{0}$ can be extended to a continuous unitary character on $Q_{f}$, it can even be extended to $Q_{g}$. The equation $x(f, \vartheta)=\left(\operatorname{Ad}^{*}(x) f, \vartheta\right)$ holds true for all $(f, \vartheta) \in \mathfrak{X}$ and all $x \in M$.

(iv) The map $J: S^{\wedge} \times M^{\wedge} \rightarrow \mathfrak{X}$ defined by $J(\sigma, \mu)=(f, \vartheta)$ where $\left.f\right|_{\mathfrak{s}}=d \sigma$, $\left.f\right|_{\mathfrak{m}}=d \mu$, and $\vartheta(s x)=\sigma(s) \mu(x)$ for $s \in S_{f}, x \in M_{f}$ is continuous; the image of $J$ consists of aligned elements in $\mathfrak{X}$.

(v) The $M$-orbits in $\mathfrak{X}$ are closed. The composition $p$ of $J$ and the quotient map $\mathfrak{X} \rightarrow \mathfrak{X} / M$ is surjective and open, the equivalence relation defined by $p$ is just $\sim_{M}$. Therefore, $J$ induces a homeomorphism from $\left(S^{\wedge} \times M^{\wedge}\right) / \sim_{M}$ onto the orbit space $\mathfrak{X} / M$. The inverse of this homeomorphism is obtained as follows. If $(f, \vartheta) \in \mathfrak{X}$ is given define $\mu \in M^{\wedge}$ by $\mu(\exp X)=e^{i f(X)}$, choose any $\sigma \in S^{\wedge}$ with $\left.\sigma\right|_{S_{f}}=\left.\vartheta\right|_{S_{f}}$ and associate with $(f, \vartheta)$ the $\sim_{M}$ equivalence class through $(\sigma, \mu)$; this map yields the desired inverse.

(vi) The map $J$ induces a homeomorphism from $\left(S^{\wedge} \times M^{\wedge}\right) / \sim$ onto $\mathfrak{X} / Q$.

Remark 1.16. Suppose that all stabilizers $Q_{f}, f \in \mathfrak{q}_{\text {int }}^{*}$, are connected as it happens for instance in the case of the full diamond group, i.e., $Q=G / Z N=U \ltimes N / Z N$. Then the second component of an element $(f, \vartheta) \in \mathfrak{X}$ is completely determined by the first one, and $\mathfrak{X} / Q$ is canonically homeomorphic to the quasi-orbit space $\mathfrak{q}_{\mathrm{int}}^{*} / Q$. More generally, by forgetting the second component $\vartheta$ the map $J$ gives a map from $S^{\wedge} \times M^{\wedge}$ into $\mathfrak{q}_{\text {int }}^{*}$, which followed by the projection onto the quasi-orbit space $\mathfrak{q}_{\text {int }}^{*} / Q$ yields an open continuous surjection $\boldsymbol{J}^{\prime}: S^{\wedge} \times M^{\wedge} \rightarrow \mathfrak{q}^{*} / Q$. Two images $\boldsymbol{J}^{\prime}(\sigma, \mu)$ and $\boldsymbol{J}^{\prime}\left(\sigma^{\prime}, \mu^{\prime}\right)$ coincide if and only if $(S \mu)^{-}=\left(S \mu^{\prime}\right)^{-}$and $\left.\sigma\right|_{\left(S_{\mu}\right)_{0}}=\left.\sigma^{\prime}\right|_{\left(S_{\mu}\right)_{0}}$. This defines another (open) equivalence relation $\stackrel{c}{\sim}$ on $S^{\wedge} \times M^{\wedge}$.

Proof. Evidently, $M$ is contained in $Q_{g}$ which implies $Q_{g}=S_{g} \ltimes M$. Together with the obvious equation $S_{g}=S_{f}$ this gives $Q_{f}=S_{f} \ltimes M_{f}$. Concerning the connectivity of $M_{f}$ one observes that any $x_{0} \in M$ can uniquely be written as $x_{0}=\exp X_{0}, X_{0} \in \mathfrak{m}$, and that $\left(\operatorname{Ad}^{*}\left(x_{0}\right) f\right)(A+X)=f(A+X)+$ $f\left(\left[A, X_{0}\right]\right)$ for all $A \in \mathfrak{s}$ and $X \in \mathfrak{m}$ or, in other letters, $\operatorname{Ad}^{*}\left(x_{0}\right) f=f+$ $\operatorname{ad}^{*}\left(X_{0}\right) f$. As all $Q_{g}$ are normal (and coabelian) one has $S_{q g}=S \cap Q_{q g}=$ $S \cap q Q_{g} q^{-1}=S \cap Q_{g}=S_{g}$ for all $q \in Q$, and therefore $S_{q f}=S_{f}$. If $x \in M$ 
then $x Q_{f} x^{-1}=Q_{x f}=S_{x f} \ltimes M_{x f}$ as we have seen already. But $S_{x f}=S_{f}$ and $M_{x f}=M_{f}$ as $M$ is abelian.

Concerning (ii) we recall the well-known relation $\left(Q_{g}\right)_{0} f=f+$ $\left(\mathfrak{q} /\left(\mathfrak{m}+\mathfrak{q}_{f}\right)\right)^{*}$, which holds true in much more general circumstances. By (i) one has $\left(Q_{g}\right)_{0} f=Q_{g} f=M f$ and $\mathfrak{m}+\mathfrak{q}_{f}=\mathfrak{m}+\mathfrak{s}_{f}$.

To construct the asserted extension in (iii) choose $\sigma \in\left(S_{f}\right)^{\wedge}$ with $\left.\sigma\right|_{\left(S_{f}\right)_{0}}=\left.\chi_{f}\right|_{\left(S_{f}\right)_{0}}$ and define $\eta: S_{f} \ltimes M \rightarrow \mathrm{T}$ by $\eta(s \exp X)=\sigma(s) e^{i f(X)}$ for $s \in S_{f}$ and $X \in \mathfrak{m}$. It is easy to check that $\eta$ is a homomorphism.

Also the second assertion in (iii) is easy as well as claim (iv).

To see that $M$-orbits in $\mathfrak{X}$ are closed, in view of (iii) it is sufficient to show that $M$-orbits in $\mathfrak{q}_{\text {int }}^{*}$ are closed which readily follows from the structure of $\operatorname{Ad}^{*}\left(x_{0}\right), x_{0} \in M$, given above; in fact $\operatorname{Ad}^{*}(M) f$ is an affine subspace. Most of the rest of (v) follows easily from (ii) and (iii), the only less obvious point is the openness of $p$. To this end, let $\left(f_{k}, \vartheta_{k}\right)$ be a convergent sequence in $\mathfrak{X}$ with limit $(f, \vartheta)$. W.1.o.g. we may assume that $(f, \vartheta)$ is aligned, i.e., $(f, \vartheta)=J(\sigma, \mu)$ with $(\sigma, \mu) \in S^{\wedge} \times M^{\wedge}$. In view of (1.9) it suffices to show that there exists a subsequence $\left(f_{k_{j}}, \vartheta_{k_{j}}\right)$ and a convergent sequence $\left(\sigma_{j}, \mu_{j}\right)$ in $S^{\wedge} \times M^{\wedge}$ with limit $(\sigma, \mu)$ such that $J\left(\sigma_{j}, \mu_{j}\right) \in M\left(f_{k_{j}}, \vartheta_{k_{j}}\right)$.

Consider the stabilizers $S_{k}:=S_{f_{k}}$ of $f_{k}$ in $S$ as a sequence in $\Sigma(S)$, which denotes the compact space of closed subgroups of $S$, compare [7,8,17]. Passing to a subsequence if necessary we may assume w.l.o.g. that $\left(S_{k}\right)$ converges to the closed subgroup $S^{\prime}$ of $S$ which by the continuity of the $S$-action has to be contained in $S_{f}$. Apply [17, Remark 2.6] to the sequence $\left(S_{k},\left.\vartheta_{k}\right|_{S_{k}}\right)$ in the space of all pairs $\left\{(H, \chi) \mid H \in \Sigma(S), \chi \in H^{\wedge}\right\}$. This sequence converges to $\left(S^{\prime},\left.\vartheta\right|_{S^{\prime}}\right)$. To the above extension $\sigma \in S^{\wedge}$ of $\left.\vartheta\right|_{S^{\prime}}$ there exist a subsequence $\left(S_{k_{j}},\left.\vartheta_{k_{j}}\right|_{S_{k_{j}}}\right)$ and extensions $\sigma_{j} \in S^{\wedge}$ of $\left.\vartheta_{k_{j}}\right|_{S_{k_{j}}}$ which converge to $\sigma$. Clearly, $\mu_{j}$ is defined by $\mu_{j}(\exp X)=e^{i f_{k_{j}}(X)}$ for $X \in \mathfrak{m}$. Then $\left(\sigma_{j}, \mu_{j}\right)$ converges to $(\sigma, \mu)$ because $\left.f_{k_{j}}\right|_{\mathfrak{m}}$ converges to $\left.f\right|_{\mathfrak{m}}$, and $J\left(\sigma_{j}, \mu_{j}\right) \in M\left(f_{k_{j}}, \vartheta_{k_{j}}\right)$ as desired.

Claim (vi) is an easy consequence of (v). The first part of the remark follows immediately from the description of the stabilizers $Q_{f}$ given in (i), which implies that in case of connected stabilizers already the spaces $\mathfrak{X}$ and $\mathfrak{q}_{\text {int }}^{*}$ are homeomorphic. For the second part observe that the canonical map $\mathfrak{X} \rightarrow \mathfrak{q}_{\text {int }}^{*}$, which is surjective by (iii), is also open, which can be proved by similar arguments as in the proof of (v), namely exploiting [17, Remark 2.6].

REMARK 1.17. The set of Q-quasi-orbits in $\mathfrak{X}$ can be used to parametrize the set of primitive ideals in $C^{*}(Q)$. This is a special case of a much more general theorem, see [19], mentioned in the introduction. On the other hand the space Priv $C^{*}(Q)$ is homeomorphic to $\left(S^{\wedge} \times M^{\wedge}\right) / \sim$ see [17] or [22]. Thus the above lemma says among others that indeed $\operatorname{Priv} C^{*}(Q)$ is homeomorphic to the $Q$ - 
quasi-orbit space $\mathfrak{X} / Q$. The only (evident) thing to be added is that the two different parametrizations fit together by means of the above induced map $\left(S^{\wedge} \times M^{\wedge}\right) / \sim \rightarrow \mathfrak{X} / Q$.

In the next proposition we consider the space of quasi-orbits on the linear dual of a "diamond algebra" $\mathfrak{s} \ltimes \mathfrak{n}$. The linear functionals on $\mathfrak{s} \ltimes \mathfrak{n}$ decompose into two classes, those which are zero on $\mathfrak{z} \mathfrak{n}(=$ center of $\mathfrak{n})$ and which were considered in Lemma 1.15, and the rest. The quasi-orbits in the rest can also be easily parametrized, and we describe the topology in terms of the parameters.

Proposition 1.18. Let $S \ltimes N$ be a diamond group, $S$ connected Lie, with Lie algebra $\mathfrak{s} \ltimes \mathfrak{n}$. Denoting by $\mathbf{R}^{\times}$the set of non-zero real numbers the set of $S \ltimes N$-quasi-orbits in $(\mathfrak{s} \ltimes \mathfrak{n})_{\text {int }}^{*}$ can be identified with $\left(S^{\wedge} \times(N / Z N)^{\wedge}\right) /$ $\stackrel{c}{\sim} \cup\left(S^{\wedge} \times \mathbf{R}^{\times}\right)$where to the $\stackrel{c}{\sim}$ equivalence class $[\sigma, \gamma]$ through $(\sigma, \gamma) \in S^{\wedge} \times$ $(N / Z N)^{\wedge}$ in the sense of (1.16) corresponds the quasi-orbit through $J(\sigma, \gamma)$ as in (1.15. iv), and to $(\sigma, \lambda) \in S^{\wedge} \times \mathrm{R}^{\times}$corresponds the $N$-orbit through $f_{\sigma, \lambda} \in(\mathfrak{s} \ltimes \mathfrak{n})^{*}$ where $f_{\sigma, \lambda}$ is defined by $f_{\sigma, \lambda}(A, z, t)=d \sigma(A)+\lambda t$ for $A \in \mathfrak{s}$, $z \in \mathrm{C}^{n}, t \in \mathrm{R}$. Transferring the topology on $(\mathfrak{s} \ltimes \mathfrak{n})_{\text {int }}^{*} / S \ltimes N$ yields a topology on $\left.S^{\wedge} \times(N / Z N)^{\wedge}\right) / \stackrel{c}{\sim} \cup\left(S^{\wedge} \times \mathbf{R}^{\times}\right)$which has the following properties:

(i) The subset $\left(S^{\wedge} \times(N / Z N)^{\wedge}\right) / \stackrel{c}{\sim}$ is closed and carries the quotient topology.

(ii) The subset $S^{\wedge} \times \mathrm{R}^{\times}$is open and carries the product topology.

(iii) A sequence $\left(\sigma_{k}, \lambda_{k}\right) \in S^{\wedge} \times \mathrm{R}^{\times}$converges to the equivalence class $[\sigma, \gamma] \in\left(S^{\wedge} \times(N / Z N)^{\wedge}\right) / \stackrel{\mathcal{c}}{\sim}$ if and only if $\left(\lambda_{k}\right)$ converges to zero and if there exists a sequence $w_{k} \in \mathrm{C}^{n}, \quad w_{k}=\left(w_{k}^{(1)}, \ldots, w_{k}^{(n)}\right)$ converging to $\gamma^{\prime}=$ $\left(\gamma_{1}, \ldots, \gamma_{n}\right) \in \mathrm{C}^{n}$ determined by $d \gamma(z)=\operatorname{Im} \gamma^{\prime} \bar{z}$ for all $z \in \mathrm{C}^{n}$, such that

$$
d \sigma=\lim _{k \rightarrow \infty} d \sigma_{k}-\frac{1}{2 \lambda_{k}} \sum_{j=1}^{n}\left|w_{k}^{(j)}\right|^{2} d \alpha_{j} .
$$

In the special case of the full diamond group $G=U \ltimes N$ the convergence criterion simplifies as follows:

(iv) Let $\left(\sigma_{k}, \lambda_{k}\right),[\sigma, \gamma]$ and $\gamma^{\prime}$ be as in (iii). Since $\zeta_{1}, \ldots, \zeta_{n}$ is a set of free generators of $U^{\wedge}$ there is a uniquely determined sequence $m_{k} \in \mathbf{Z}^{n}$, $m_{k}=\left(m_{k}^{(1)}, \ldots, m_{k}^{(n)}\right)$, such that

$$
\sigma_{k}=\sigma \prod_{j=1}^{n} \zeta_{j}^{m_{k}^{(j)}} \text { or } \quad d \sigma_{k}=d \sigma+\sum_{j=1}^{n} m_{k}^{(j)} d \zeta_{j}
$$

The sequence $\left(\sigma_{k}, \lambda_{k}\right)$ converges to $[\sigma, \gamma]$ if and only if $\left(\lambda_{k}\right)$ converges to zero, if $\lim _{k \rightarrow \infty} 2 \lambda_{k} m_{k}^{(j)}=\left|\gamma_{j}\right|^{2}$ for $1 \leq j \leq n$, and if there is an $k_{0}$ such that $\lambda_{k} m_{k}^{(j)} \geq 0$ for all $k \geq k_{0}$ and all $j$ (which is of course only relevant if $\gamma_{j}=0$ for some $j$ ). 
ReMARK 1.19. I don't know in general how to express the criterion in (iii) directly in terms of $\left(\sigma_{k}, \lambda_{k}\right)$ and $(\sigma, \gamma)$. Clearly, the criterion implies that $2 \lambda_{k}\left(d \sigma_{k}-d \sigma\right)$ and $2 \lambda_{k} d \sigma_{k}$ converge to $\sum_{j=1}^{n}\left|\gamma_{j}\right|^{2} d \alpha_{j}$, but this condition is not sufficient.

Proof. Consider more general than $f_{\sigma, \lambda}$ functionals $f_{h, \lambda}, h \in \mathfrak{s}^{*}, \lambda \neq 0$, given by

$$
f_{h, \lambda}(A, z, t)=h(A)+\lambda t .
$$

Those functionals are fixed by $S$, hence their $S \ltimes N$-orbit coincides with the $N$-orbit. A general element in $N$ can be written as $\left(\frac{1}{\lambda} w_{0}, t_{0}\right), t_{0} \in \mathrm{R}$, $w_{0}=\left(w_{0}^{(1)}, \ldots, w_{0}^{(n)}\right) \in \mathrm{C}^{n}$. Applying such an element to $f_{h, \lambda}$ yields a functional $f^{\prime}$ on $\mathfrak{s} \ltimes \mathfrak{n}$, namely:

$$
\begin{aligned}
f^{\prime}(A, z, t) & =f_{h, \lambda}\left(\operatorname{Ad}\left(\frac{1}{\lambda} w_{0}, t_{0}\right)^{-1}(A, z, t)\right) \\
& =f_{h, \lambda}\left(A, z^{\prime}, t+\frac{1}{\lambda} \operatorname{Im} w_{o} \bar{z}-\frac{1}{2 \lambda^{2}} \sum_{j=1}^{n} d \alpha_{j}(A)\left|w_{0}^{(j)}\right|^{2}\right)
\end{aligned}
$$

by (1.8) for some $z^{\prime} \in \mathrm{C}^{n}$ which doesn't matter, hence

$$
f^{\prime}(A, z, t)=h(A)-\frac{1}{2 \lambda} \sum_{j=1}^{n}\left|w_{0}^{(j)}\right|^{2} d \alpha_{j}(A)+\lambda t+\operatorname{Im}\left(w_{0} \bar{z}\right) .
$$

This shows that the $(S \ltimes N)$-orbit through $f_{h, \lambda}$ is closed, and it puts in evidence that any functional $f$ on $\mathfrak{s} \ltimes \mathfrak{n}$ with $f(\mathfrak{z} \mathfrak{n}) \neq 0$ is $S \ltimes N$ - or $N$-conjugate to an $f_{h, \lambda}$ with uniquely determined $h$ and $\lambda$ (and $w_{0}$ ). If $f$ is integrable then the corresponding $f_{h, \lambda}$ is integrable as well, which means, as $S \times Z N$ is the stabilizer of $f_{h, \lambda}$, that $h$ is the differential of some $\sigma \in S^{\wedge}$. We conclude that the $S \ltimes N$-quasi-orbit through integrable functionals in general position are actually closed $N$-orbits, and that the set of those quasi-orbits can be parametrized by $S^{\wedge} \times \mathrm{R}^{\times}$as claimed in the proposition.

Concerning the topology claim (i) is clear in view of (1.16). To see that $S^{\wedge} \times \mathbf{R}^{\times}$carries the product topology take a sequence $\left(\sigma_{k}, \lambda_{k}\right)$ such that the $N$-orbits through $f_{\sigma_{k}, \lambda_{k}}$ converge to the $N$-orbit through $f_{\sigma, \lambda}$ for some real $\lambda \neq 0$ and $\sigma \in S^{\wedge}$. This is equivalent to the existence of a sequence $w_{k} \in \mathrm{C}^{n}$ such that the sequence $f_{k}^{\prime}$ defined by

$$
f_{k}^{\prime}(A, z, t)=d \sigma_{k}(A)-\frac{1}{2 \lambda_{k}} \sum_{j=1}^{n}\left|w_{k}^{(j)}\right|^{2} d \alpha_{j}(A)+\lambda_{k} t+\operatorname{Im}\left(w_{k} \bar{z}\right)
$$


converges to $f_{\sigma, \lambda}$. Then necessarily $\left(\lambda_{k}\right)$ converges to $\lambda$ and $\left(w_{k}\right)$ converges to 0 , whence $\left(d \sigma_{k}\right)$ converges to $d \sigma$.

Similarly claim (iii) follows from the description of the $\mathrm{N}$-orbits through $f_{\sigma_{k}, \lambda_{k}}$.

Concerning claim (iv) suppose first that the convergence criterion is satisfied. We wish to apply (iii), and thus we have to specify a sequence $\left(w_{k}\right)$ in $\mathrm{C}^{n}$ as there. For $k \geq k_{0}$ define $w_{k}^{(j)}=\left(2 \lambda_{k} m_{k}^{(j)}\right)^{\frac{1}{2}}$ if $\gamma_{j}=0$, and $w_{k}^{(j)}=$ $\left(2 \lambda_{k} m_{k}^{(j)}\right)^{\frac{1}{2}} \frac{\gamma_{j}}{\left|\gamma_{j}\right|}$ if $\gamma_{j} \neq 0$. It is trivial to check that the criterion of (iii) is satisfied, one even has

$$
d \sigma=d \sigma_{k}-\frac{1}{2 \lambda_{k}}\left|w_{k}^{(j)}\right|^{2} d \zeta_{j}
$$

for all $k \geq k_{0}$.

For the other implication we apply (iii) once more, and find a sequence $\left(w_{k}\right)$ in $\mathrm{C}^{n}$ converging to $\left(\gamma_{1}, \ldots, \gamma_{n}\right)$ with

$$
d \sigma=\lim _{k \rightarrow \infty} d \sigma_{k}-\frac{1}{2 \lambda_{k}} \sum_{j=1}^{n}\left|w_{k}^{(j)}\right|^{2} d \zeta_{j}
$$

Inserting $d \sigma_{k}=d \sigma+\sum_{j=1}^{n} m_{k}^{(j)} d \zeta_{j}$ yields

$$
0=\lim _{k \rightarrow \infty} \sum_{j=1}^{n}\left(m_{k}^{(j)}-\frac{1}{2 \lambda_{k}}\left|w_{k}^{(j)}\right|^{2}\right) d \zeta_{j}
$$

whence for each $j$ the sequence $\varepsilon_{k}^{(j)}:=m_{k}^{(j)}-\frac{1}{2 \lambda_{k}}\left|w_{k}^{(j)}\right|^{2}$ converges to zero as $k$ tends to infinity. Multiplying by $2 \lambda_{k}$ yields that $\left(2 \lambda_{k} m_{k}^{(j)}\right)$ converges to $\lim _{k \rightarrow \infty}\left|w_{k}^{(j)}\right|^{2}=\left|\gamma_{j}\right|^{2}$.

It remains to consider that $\gamma_{j}=0$ for some $j$. From the definition of $\varepsilon_{k}^{(j)}$ it follows that

$$
0 \leq \frac{\left|w_{k}^{(j)}\right|^{2}}{\left|2 \lambda_{k}\right|}=\frac{\lambda_{k}}{\left|\lambda_{k}\right|} m_{k}^{(j)}-\frac{\lambda_{k}}{\left|\lambda_{k}\right|} \varepsilon_{k}^{(j)} .
$$

As $\frac{\lambda_{k}}{\left|\lambda_{k}\right|} \varepsilon_{k}^{(j)}$ tends to zero and as $\frac{\lambda_{k}}{\left|\lambda_{k}\right|} m_{k}^{(j)}$ is an integer one concludes $\lambda_{k} m_{k}^{(j)} \geq 0$ for sufficiently large $k$.

Part (v) of Lemma 1.15 and our above arguments also give a description of the space of $N$-orbits in $\mathfrak{g}_{\mathrm{int}}^{*}$. Let $\mathfrak{Y}$ be the disjoint union of $U^{\wedge} \times \mathrm{R}^{\times}$and $U^{\wedge} \times(N / Z N)^{\wedge}$. Define a (non-Hausdorff) topology on $\mathfrak{Y}$ by requiring that $U^{\wedge} \times \mathbf{R}^{\times}$is open in $\mathfrak{Y}$, and that $W_{\varepsilon}(\omega, \gamma), \varepsilon>0$, is a neighborhood basis of $(\omega, \gamma) \in U^{\wedge} \times(N / Z N)^{\wedge}$ where $W_{\varepsilon}(\omega, \gamma)$ is the union of the set of all pairs $(\omega, \mu) \in U^{\wedge} \times(N / Z N)^{\wedge}$ such that $\left|\mu_{j}-\gamma_{j}\right|<\varepsilon$ for $1 \leq j \leq n$ if $d \gamma(z)=$ 
$\operatorname{Im} \sum_{j=1}^{n} \gamma_{j} \overline{z_{j}}$ and $d \mu(z)=\operatorname{Im} \sum_{j=1}^{n} \mu_{j} \overline{z_{j}}$ for $z \in \mathrm{C}^{n}$, and of the set of all pairs $\left(\omega^{\prime}, \lambda\right) \in U^{\wedge} \times \mathrm{R}^{\times}$such that $|\lambda|<\varepsilon, d \omega^{\prime}=d \omega+\sum_{j=1}^{n} m_{j} d \zeta_{j}, m_{j} \lambda \geq 0$ and $\left|\left(2 m_{j} \lambda\right)^{\frac{1}{2}}-\right| \gamma_{j}||<\varepsilon$ for $1 \leq j \leq n$.

Remark 1.21. A sequence $\left(\omega_{k}, \lambda_{k}\right) \in U^{\wedge} \times \mathrm{R}^{\times}$converges to $(\omega, \gamma) \in U^{\wedge} \times$ $(N / Z N)^{\wedge}$ w.r.t. the topology of $\mathfrak{Y}$ if and only if $\left(\lambda_{k}\right)$ converges to zero, $\lambda_{k} m_{k}^{(j)} \geq 0$ for all sufficiently large $k$, and $\left(2 \lambda_{k} m_{k}^{(j)}\right)$ converges to $\left|\gamma_{j}\right|^{2}$ for $1 \leq j \leq n$ where $d \gamma(z)=\operatorname{Im} \sum_{j=1}^{n} \gamma_{j} \bar{z}_{j}$ for $z \in \mathrm{C}^{n}$, and where $m_{k}^{(j)}$ is defined by the equation

$$
d \omega_{k}=d \omega+\sum_{j=1}^{n} m_{k}^{(j)} d \zeta_{j}
$$

The (discontinuous) map $J: \mathfrak{Y} \rightarrow \mathfrak{g}_{\mathrm{int}}^{*}$ defined on $U^{\wedge} \times(N / Z N)^{\wedge}$ as in Lemma 1.15 (iv), i.e. $J(\omega, \gamma)=d \omega+d \gamma$, and on $U^{\wedge} \times \mathrm{R}^{\times}$by $J(\omega, \lambda)=f_{\omega, \lambda}$ where $f_{\omega, \lambda}$ is as in the proof of Proposition 1.18, followed by the quotient map $\mathfrak{g}_{\mathrm{int}}^{*} \rightarrow \mathfrak{g}_{\mathrm{int}}^{*} / N$ is continuous, open and onto. The equivalence relation $\sim_{\mathfrak{Y}}$ defined by this map is the identity on $U^{\wedge} \times \mathbf{R}^{\times}$, and $(\omega, \gamma)$ and $\left(\omega^{\prime}, \gamma^{\prime}\right)$ in $U^{\wedge} \times(N / Z N)^{\wedge}$ are equivalent if and only if $\gamma=\gamma^{\prime}$ and $\omega=\omega^{\prime}$ on the stabilizer $U_{\gamma}$. The space $\mathfrak{g}_{\mathrm{int}}^{*} / N$ is homeomorphic to $\mathfrak{Y} / \sim_{\mathfrak{Y}}$.

\section{§. The Unitary Dual of the Full Diamond Group}

In this section we describe all irreducible unitary representations of the full diamond group $G=U \ltimes N$ up to unitary equivalence. In contrast to more general diamond groups the full diamond group is of type I. It turns out that the unitary dual $G^{\wedge}$ is homeomorphic to the $G$-orbit space $\mathfrak{g}_{\mathrm{int}}^{*} / G$.

In order not to interrupt the discussion at a later point we start with a lemma on one of the Bessel functions, which will be the basic analytic tool for proving the result on the dual topology. Recall that the Bessel function $J_{0}$ has the power series expansion

$$
J_{0}(z)=\sum_{j=0}^{\infty}(-1)^{j} \frac{1}{(j !)^{2}}\left(\frac{z}{2}\right)^{2 j}
$$

compare e.g. [21, p. 358].

LeMma 2.1. For a sequence $\left(a_{k}\right)$ of positive real numbers converging to zero and for a sequence $\left(m_{k}\right)$ of nonnegative integers form the (even) real polynomial functions

$$
\Phi_{k}(x)=\sum_{j=0}^{m_{k}}(-1)^{j} \frac{1}{j !}\left(\begin{array}{c}
m_{k} \\
j
\end{array}\right)\left(\frac{a_{k}}{2}\right)^{j} x^{2 j}
$$


(1) If $\left(2 a_{k} m_{k}\right)$ converges to $b^{2}$ for some $b>0$ then $\left(\Phi_{k}\right)$ converges uniformly on compacta to the function $x \mapsto J_{0}(b x)$.

(2) If $\left(a_{k} m_{k}\right)$ converges to $\infty$ then $\left(\Phi_{k}\right)$ does not converge uniformly on compacta.

(3) If $\left(a_{k} m_{k}\right)$ converges to zero then $\left(\Phi_{k}\right)$ converges to 1 uniformly on compacta.

Proof. Put $b_{k}^{2}=2 a_{k} m_{k}$ with $b_{k} \geq 0$. In the first case $\left(b_{k}\right)$ converges to $b$, and $\left(m_{k}\right)$ converges to $\infty$. Let $\varepsilon>0$ and $R>0$ be given. Choose $R^{\prime}$ such that $b_{k} R \leq R^{\prime}$ for all $k$, and $M \in \mathrm{N}$ such that

$$
\sum_{j=M+1}^{\infty}\left(\frac{1}{j !}\right)^{2}\left(\frac{R^{\prime}}{2}\right)^{2 j}<\varepsilon .
$$

For sufficiently large indices $k$ one has $m_{k}>M$. Substituting $a_{k}$ by $\frac{1}{2 m_{k}} b_{k}^{2}$ leads to

$$
\begin{aligned}
\Phi_{k}(x) & =\sum_{j=0}^{m_{k}}(-1)^{j} \frac{1}{j !}\left(\begin{array}{c}
m_{k} \\
j
\end{array}\right)\left(\frac{b_{k}^{2}}{4 m_{k}}\right)^{j} x^{2 j} \\
& =\sum_{j=0}^{m_{k}}(-1)^{j}\left(\frac{1}{j !}\right)^{2} \frac{m_{k}\left(m_{k}-1\right) \cdot \ldots \cdot\left(m_{k}-j+1\right)}{m_{k}^{j}}\left(\frac{b_{k} x}{2}\right)^{2 j} .
\end{aligned}
$$

Split this sum into $\sum_{j=0}^{M}+\sum_{j=M+1}^{m_{k}}$. The absolute value of the second summand is less than $\varepsilon$ for $|x| \leq R$. The first summand converges uniformly in $|x| \leq R$ to

$$
\sum_{j=0}^{M}(-1)^{j}\left(\frac{1}{j !}\right)^{2}\left(\frac{b x}{2}\right)^{2 j}
$$

because $\left(m_{k}\right)$ tends to infinity; and this is the $M^{\text {th }}$ partial sum of the power series expansion of $x \mapsto J_{0}(b x)$. Again the absolute value of the rest of the latter series is estimated by $\varepsilon$ in $|x| \leq R$.

In the second case put $a_{k}^{\prime}:=\frac{1}{2 m_{k}}$ and form also the $\Phi$-functions to the parameters $a_{k}^{\prime}$ and $m_{k}$, i.e.,

$$
\Psi_{k}(x):=\sum_{j=0}^{m_{k}}(-1)^{j} \frac{1}{j !}\left(\begin{array}{c}
m_{k} \\
j
\end{array}\right)\left(\frac{1}{4 m_{k}}\right)^{j} x^{2 j} .
$$

As we have seen in the first case the sequence $\left(\Psi_{k}\right)$ converges to $J_{0}$ uniformly on compacta. For each $x$ put $x_{k}=\frac{x}{b_{k}}$, which converges to zero by assumption. Furthermore, one has

$$
\Phi_{k}\left(x_{k}\right)=\Psi_{k}(x)
$$


for all $x \in \mathrm{R}$. If $\left(\Phi_{k}\right)$ would converge uniformly on compacta to $\Phi_{\infty}$, say, then for each $x$ the sequence $\Phi_{k}\left(x_{k}\right)=\Psi_{k}(x)$ would converge to $\Phi_{\infty}(0)=J_{0}(x)$, which is impossible.

In the third case we may assume $0 \leq b_{k} \leq 1$. Let again $\varepsilon, R>0$ be given, and choose $M \in \mathrm{N}$ such that

$$
\sum_{j=M+1}^{\infty}\left(\frac{1}{j !}\right)^{2} j\left(\frac{R}{2}\right)^{2 j}<\varepsilon .
$$

For all $x$ one has

$$
\begin{aligned}
\left|\Phi_{k}(x)-1\right| & =\left|\sum_{j=1}^{m_{k}}(-1)^{j}\left(\frac{1}{j !}\right)^{2} \frac{m_{k} \cdot \ldots \cdot\left(m_{k}-j+1\right)}{m_{k}^{j}}\left(\frac{b_{k} x}{2}\right)^{2 j}\right| \\
& \leq \sum_{j=1}^{\infty}\left(\frac{1}{j !}\right)^{2}\left(\frac{b_{k} x}{2}\right)^{2 j}=\sum_{j=1}^{M}\left(\frac{1}{j !}\right)^{2}\left(\frac{b_{k} x}{2}\right)^{2 j}+\sum_{j=M+1}^{\infty}\left(\frac{1}{j !}\right)^{2}\left(\frac{b_{k} x}{2}\right)^{2 j} .
\end{aligned}
$$

If $|x| \leq R$ the second summand is smaller than $\varepsilon$ while the first one tends to zero uniformly as $\left(b_{k}\right)$ converges to zero.

The irreducible unitary representations of $G$ are divided into two classes, into those $\pi$ with $\pi(1,0, t)=e^{i \lambda t} \mathrm{Id}$ for some $\lambda \in \mathrm{R}^{\times}$and all $(1,0, t) \in Z G$, and into those which are trivial on $Z G$. By Mackey analysis the latter ones can be parametrized by $(\omega, \gamma) \in U^{\wedge} \times(N / Z N)^{\wedge}$ as follows, compare also [17, Theorem 3.7]. Define $\pi_{\omega, \gamma}$ in $L^{2}\left(U / U_{\gamma}\right)$ by

$$
\left(\pi_{\omega, \gamma}(s, z, t) \xi\right)(u)=\omega(s) \gamma\left(\zeta\left(u^{-1} s\right)(z)\right) \xi\left(s^{-1} u\right)
$$

for $s \in U, z \in \mathrm{C}^{n}, t \in \mathrm{R}, u \in U / U_{\gamma}$ and $\xi \in L^{2}\left(U / U_{\gamma}\right)$, where $\gamma \in(N / Z N)^{\wedge}$ is viewed as a unitary character on $\mathrm{C}^{n}$. Two such representations $\pi_{\omega, \gamma}$ and $\pi_{\omega^{\prime}, \gamma^{\prime}}$ are unitarily equivalent if and only if $(\omega, \gamma) \sim\left(\omega^{\prime}, \gamma^{\prime}\right)$ for the equivalence relation $\sim$ defined in (1.11), which in the case at hand coincides with $\stackrel{c}{\sim}$ of (1.16). To an equivalence class through $(\omega, \gamma)$ corresponds by (1.18) a $G$ orbit in $\mathfrak{g}_{\text {int }}^{*}$, and clearly $\pi_{\omega, \gamma}$ is nothing but the representation attached to this orbit.

To describe the representations in general position we first recall the complex realization of the infinite-dimensional representations of the Heisenberg group, compare e.g. [9], even though our notations differ from those used there. The main difference is that all our representations are realized in spaces of holomorphic functions, never do antiholomorphic functions appear. This is to reflect that the general procedure of "holomorphic induction", as created in [1] and described in book form in [2], always leads to conditions of holomorphy. On the other hand we do not deal with general 
positive polarizations subordinate to arbitrary functionals, but rather work with functionals adapted to the chosen "coordinate system" which in particular means that those are stabilized by $U$ in the fixed, but not canonical decomposition $G=U \ltimes N$.

For real $\lambda \neq 0$ let $\mathfrak{F}_{\lambda}$ be the Hilbert space of all entire functions $\xi: \mathrm{C}^{n} \rightarrow \mathrm{C}$ with

$$
\|\xi\|^{2}=\int_{\mathrm{C}^{n}}|\xi(w)|^{2} e^{-\frac{1}{2}|\lambda||w|^{2}} d w<\infty .
$$

Define an irreducible unitary representation $\pi_{\lambda}$ of $N$ in $\mathfrak{F}_{\lambda}$ by

$$
\left(\pi_{\lambda}(z, t) \xi\right)(w)=e^{i \lambda t-\frac{1}{2} \lambda w \bar{z}-\frac{1}{4}|z|^{2} \lambda} \xi(w+z) \text { if } \lambda>0
$$

and by

$$
\left(\pi_{\lambda}(z, t) \xi\right)(w)=e^{i \lambda t+\frac{1}{2} \lambda w z+\frac{1}{4} \lambda|z|^{2}} \xi(w+\bar{z}) \text { if } \lambda<0,
$$

where $z, w \in \mathbf{C}^{n}, t \in \mathbf{R}$ and $\xi \in \mathfrak{F}_{\lambda}$.

For $s \in U$ define a unitary operator $K_{\lambda}(s)$ in $\mathfrak{F}_{\lambda}$ by

$$
\left(K_{\lambda}(s) \xi\right)(w)=\xi\left(\zeta(s)^{-1} w\right) \text { if } \lambda>0
$$

and by

$$
\left(K_{\lambda}(s) \xi\right)(w)=\xi(\zeta(s) w) \text { if } \lambda<0
$$

where $\zeta(s) w \in \mathrm{C}^{n}=N / Z N$ has the obvious meaning. For each pair $(\omega, \lambda)$, $\omega \in U^{\wedge}, \quad \lambda \neq 0$ define a representation $\pi_{\omega, \lambda}$ of $G=U \ltimes N$ in $\mathfrak{F}_{\lambda}$ by $\left.\pi_{\omega, \lambda}\right|_{N}=\pi_{\lambda}$ and $\pi_{\omega, \lambda}(s, 0,0)=\omega(s) K_{\lambda}(s)$ for $s \in U$. It is easy to see that $\pi_{\omega, \lambda}$ is well-defined. Explicitly, one has

$$
\left(\pi_{\omega, \lambda}(s, z, t) \xi\right)(w)=\omega(s) e^{i \lambda t-\frac{1}{2} \lambda\left(\zeta\left(s^{-1}\right) w\right) \bar{z}-\frac{1}{4} \lambda|z|^{2}} \xi\left(z+\zeta\left(s^{-1}\right) w\right) \quad \text { if } \quad \lambda>0
$$

and

$$
\left.\left(\pi_{\omega, \lambda}(s, z, t) \xi\right) w\right)=\omega(s) e^{i \lambda t+\frac{1}{2} \lambda(\zeta(s) w) z+\frac{1}{4} \lambda|z|^{2}} \xi(\zeta(s) w+\bar{z}) \text { if } \lambda<0 .
$$

Each irreducible unitary representation of $G$ which restricts to $e^{i \lambda t}$ on $Z G$ with $\lambda \neq 0$ is unitarily equivalent to $\pi_{\omega, \lambda}$ for a uniquely determined $\omega \in U^{\wedge}$. Moreover, to the pair $(\omega, \lambda)$ corresponds a certain orbit in $\mathfrak{g}_{\mathrm{int}}^{*}$ in the sense of (1.18), and the representation (class) attached to this orbit by the general procedure is just $\pi_{\omega, \lambda}$. Altogether we obtain a bijection from the homeomorphic (by definition) spaces $\left(U^{\wedge} \times(N / Z N)^{\wedge}\right) / \sim \cup\left(U^{\wedge} \times \mathrm{R}^{\times}\right)$and $\mathfrak{g}_{\text {int }}^{*} / G$ onto the unitary dual $G^{\wedge}$.

THEOREM 2.6. The above bijection is a homeomorphism, i.e., $G^{\wedge}$ is home- 
omorphic to the orbit space $\mathfrak{g}_{\mathrm{int}}^{*} / G$. In particular, in view of (1.18. iv) if sequences $\left(\omega_{k}\right)$ in $U^{\wedge}$ and $\left(\lambda_{k}\right)$ in $\mathrm{R}^{\times}$as well as $\omega \in U^{\wedge}$ and $\gamma \in(N / Z N)^{\wedge}$, $\gamma(z, t)=e^{i \operatorname{Im}}\left(\gamma^{\prime} \bar{z}\right)$ with $\gamma^{\prime}=\left(\gamma_{1}, \ldots, \gamma_{n}\right) \in \mathbf{C}^{n}$, are given then $\pi_{\omega_{k}, \lambda_{k}}$ converges to $\pi_{\omega, \lambda}$ if and only if $\left(\lambda_{k}\right)$ converges to zero, if $\lim _{k \rightarrow \infty} 2 \lambda_{k} m_{k}^{(j)}=\left|\gamma_{j}\right|^{2}$ for $1 \leq j \leq n$ and if $\lambda_{k} m_{k}^{(j)} \geq 0$ for all $j$ and all sufficiently large $k$, where $m_{k}^{(j)} \in \mathbf{Z}$, is defined by $d \omega_{k}=d \omega+\sum_{j=1}^{n} m_{k}^{(j)} d \zeta_{j}$.

REMARK 2.7. In terms of the topological space $\mathfrak{Y}=U^{\wedge} \times \mathrm{R}^{\times} \cup U^{\wedge} \times$ $(N / Z N)^{\wedge}$ defined in front of (1.21) the theorem may be reformulated as follows. The map $\mathfrak{Y} \rightarrow G^{\wedge}$ defined by assigning to $(\omega, \mu) \in \mathfrak{Y}$, i.e., $0 \neq \mu \in \mathbf{R}$ or $\mu \in(N / Z N)^{\wedge}$, the unitary equivalence class of $\pi_{\omega, \mu}$ is open, continuous and onto.

Proof. The homeomorphy of $(G / Z N)^{\wedge}$ with $\left(U^{\wedge} \times(N / Z N)^{\wedge}\right) / \sim$ follows from [22] and [17, Theorem 3.3 and the remarks in front of Theorem 3.7]. The homeomorphy of the open subset $G^{\wedge} \backslash(G / Z N)^{\wedge}$ with $U^{\wedge} \times \mathrm{R}^{\times}$is equally easy. Suppose for instance that $\pi_{\omega_{k}, \lambda_{k}}$ converges to $\pi_{\omega, \lambda}, \lambda \neq 0$. Restricting to $Z N$ yields that $\left(\lambda_{k}\right)$ converges to $\lambda$. Next observe that the group $\mathbf{R}_{+}$of positive real numbers acts by dilations $\delta_{r}, \delta_{r}(s, z, t)=\left(s, r z, r^{2} t\right)$, continuously and automorphically on $G$, hence $\left(\mathrm{R}_{+}, G^{\wedge}\right)$ is a topological transformation group. As $\left(\lambda_{k}\right)$ converges to $\lambda$ the sequence $r_{k}:=\left(\frac{\lambda}{\lambda_{k}}\right)^{\frac{1}{2}}$ converges to 1 . Therefore, the sequence of representations $\pi_{\omega_{k}, \lambda_{k}} \circ \delta_{r_{k}}$ converges to $\pi_{\omega, \lambda}$ as $k$ tends to infinity. And it is easy to see that $\pi_{\omega_{k}, \lambda_{k}} \circ \delta_{r_{k}}$ is unitarily equivalent to $\pi_{\omega_{k}, \lambda}$, hence $\pi_{\omega_{k}, \lambda}$ tends to $\pi_{\omega, \lambda}$ as $k$ goes to infinity. But the set of representations lying over a fixed $\lambda \neq 0$ is homeomorphic to $U^{\wedge}$, cf. also Remark 3.9. Therefore, the sequence $\left(\omega_{k}\right)$ converges to $\omega$, which in the case at hand even means $\omega_{k}=\omega$ eventually.

To see the continuity of $G^{\wedge} \rightarrow \mathfrak{g}_{\text {int }}^{*} / G$ it now suffices to show that the criterion of the theorem is satisfied if the sequence $\left(\pi_{\omega_{k}, \lambda_{k}}\right)$ converges to $\pi_{\omega, \lambda}$. It even suffices to show that there exists a subsequence satisfying the criterion. Moreover, taking tensor products with $\omega^{-1}$ we may suppose that $\omega$ is the trivial character. Clearly, restricting $\pi_{\omega_{k}, \lambda_{k}}$ to $Z N$ shows that $\left(\lambda_{k}\right)$ must converge to zero. Passing to a subsequence we may suppose that all $\lambda_{k}$ have the same sign. In the following we shall only treat the case that all $\lambda_{k}$ are negative, and put $a_{k}:=-\lambda_{k}$. The assumption that $\left(\pi_{\omega_{k}, \lambda_{k}}\right)$ converges to $\pi_{\omega, \gamma}=\pi_{1, \gamma}$ means that for each matrix coefficient $\varphi$ of $\pi_{1, \gamma}, \varphi(y)=\left\langle\pi_{1, \gamma}(y) \xi, \xi\right\rangle$ where $\xi$ is a unit vector in the space $L^{2}\left(U / U_{\gamma}\right)$ of $\pi_{1, \gamma}$, there exists a sequence $\left(\xi_{k}\right)$ of unit vectors in $\mathfrak{F}_{\lambda_{k}}$ such that $\varphi_{k}, \varphi_{k}(y):=\left\langle\pi_{\omega_{k}, \lambda_{k}}(y) \xi_{k}, \xi_{k}\right\rangle$, converges on $G$ uniformly on compacta to $\varphi$ as $k$ tends to infinity. Next we specify $\xi$ to be the constant 1 and compute $\varphi$. 
For $\gamma^{\prime}=\left(\gamma_{1}, \ldots, \gamma_{n}\right)$ as in the theorem and $y=\left(s, z_{1}, \ldots, z_{n}, t\right) \in G=$ $U \ltimes N$ one has

$$
\begin{aligned}
\varphi(y) & =\int_{U / U_{\gamma}}\left(\pi_{1, \gamma}(y) \xi\right)(u) d u \\
& =\int_{U}\left(\gamma\left(\zeta\left(u^{-1} s\right)(z)\right) d u=\int_{U} \gamma\left(\zeta\left(u^{-1}\right)(z)\right) d u\right. \\
& =\int_{U} e^{i \operatorname{Im} \sum_{j=1}^{n} \gamma_{j} \zeta_{j}(u) \bar{z}_{j}} d u \\
& =\prod_{j=1}^{n} \int_{\mathbf{T}} e^{i \operatorname{Im} \gamma_{j} v \bar{z}_{j}} d v .
\end{aligned}
$$

The integrals are easily "computed". By the invariance of the Haar measure on $\mathrm{T}$ one gets

$$
\int_{\mathrm{T}} e^{i \operatorname{Im} \gamma_{j} v \bar{z} j} d v=\int_{\mathrm{T}} e^{i \operatorname{Im}\left|\gamma_{j} z_{j}\right| v} d v=\frac{1}{2 \pi} \int_{0}^{2 \pi} e^{i\left|\gamma_{j} z_{j}\right| \sin \vartheta} d \vartheta
$$

which is nothing but $J_{0}\left(\left|\gamma_{j} z_{j}\right|\right)$, compare [21, p. 362]. Thus we obtain the result

$$
\varphi\left(s, z_{1}, \ldots, z_{n}, t\right)=\prod_{j=1}^{n} J_{0}\left(\left|\gamma_{j} z_{j}\right|\right) .
$$

In particular we see that $\varphi$ is invariant under left and right translations with elements in $U$, which is not too surprising as $\xi$ is fixed by $\pi_{1, \gamma}(U)$. If the above matrix coefficients $\varphi_{k}$ converge to $\varphi$ uniformly on compacta then also their two-sided averages $\varphi_{k}^{\natural}, \varphi_{k}^{\natural}(y)=\int_{U} \int_{U} \varphi_{k}(u y v) d u d v$ will do so. But as

$$
\varphi_{k}^{\natural}(y)=\left\langle\pi_{\omega_{k}, \lambda_{k}}(y) \xi_{k}^{\natural}, \xi_{k}^{\natural}\right\rangle
$$

with $\xi_{k}^{\natural}=\int_{U} \pi_{\omega_{k}, \lambda_{k}}(u) \xi_{k} d u$, we may assume from the beginning that $\xi_{k}$ is fixed by $\pi_{\omega_{k}, \lambda_{k}}(U)$. (the norm of $\xi_{k}^{\natural}$ might be less than one, but as $\varphi_{k}^{\natural}(e)$ converges to 1 , one can renormalize for all sufficiently large $k$, hence w.o.l.g. for all $k$ as we are free to pass to subsequences.) This implies already a restriction on the sequence $\left(\omega_{k}, \lambda_{k}\right)$ because not all representations in general position do contain $U$-fixed vectors. Actually the $U$-eigenvectors for the various $\pi_{\omega, \lambda}$ in $\mathfrak{F}_{\lambda}$ are precisely the monomials, and the corresponding eigencharacters for a given $\pi_{\omega, \lambda}$ form a "discrete cone" in $U^{\wedge}$ :

For $q_{k} \in \mathrm{N}_{0}^{n}, q_{k}=\left(q_{k}^{(1)}, \ldots, q_{k}^{(n)}\right)$, denote by $P_{k}$ the corresponding monomial, $P_{k}(z)=z^{q_{k}}=z_{1}^{q_{k}^{(1)}} \cdot \ldots \cdot z_{n}^{q_{k}^{(n)}}$. As $\omega_{k}=\prod_{j=1}^{n} \zeta_{j}^{m_{k}^{(j)}}$ one has 


$$
\left(\pi_{\omega_{k}, \lambda_{k}}(s) P_{k}\right)(w)=\prod_{j=1}^{n} \zeta_{j}(s)^{m_{k}^{(j)}} \prod_{j=1}^{n}\left(\zeta_{j}(s) w_{j}\right)^{q_{k}^{(j)}}
$$

hence

$$
\pi_{\omega_{k}, \lambda_{k}}(s) P_{k}=\prod_{j=1}^{n} \zeta_{j}(s)^{m_{k}^{(j)}+q_{k}^{(j)}} P_{k}
$$

The only chance to get a $U$-fixed vector is to choose $q_{k}^{(j)}:=-m_{k}^{(j)}$. As $q_{k}^{(j)}$ has to be nonnegative the convergence of $\pi_{\omega_{k}, \lambda_{k}}$ forces $m_{k}^{(j)} \leq 0$ which together with $\lambda_{k}<0$ yields $\lambda_{k} m_{k}^{(j)} \geq 0$ as desired. Furthermore, the matrix coefficient $\varphi_{k}$ is given by

$$
\varphi_{k}(y)=c_{k}\left\langle\pi_{\omega_{k}, \lambda_{k}}(y) P_{k}, P_{k}\right\rangle
$$

with some positive constant $c_{k}$, which is determined by $\varphi_{k}(e)=1$. Next we compute this matrix coefficient $\varphi_{k}$.

By the $U$-invariance one has

$$
\begin{aligned}
\varphi_{k}(s, z, t) & =\varphi_{k}(1, z, t)=c_{k}\left\langle\pi_{\lambda_{k}}(z, t) P_{k}, P_{k}\right\rangle \\
& =c_{k} e^{i \lambda_{k} t} \prod_{j=1}^{n} \int_{C} e^{\frac{1}{2} \lambda_{k} w z_{j}+\frac{1}{4} \lambda_{k}\left|z_{j}\right|^{2}}\left(w+\bar{z}_{j}\right)^{q_{k}^{(j)}} \bar{w}^{q_{k}^{(j)}} e^{-\frac{1}{2} a_{k}|w|^{2}} d w ;
\end{aligned}
$$

recall that $a_{k}=-\lambda_{k}=\left|\lambda_{k}\right|$. Each of the integrals is easily computed. Writing $a, z$ and $q$ instead of $a_{k}, z_{j}$ and $q_{k}^{(j)}$ for the moment we have to consider

$$
\int_{\mathrm{C}} e^{-\frac{1}{2} a w z-\frac{1}{4} a|z|^{2}}(w+\bar{z})^{q} \bar{w}^{q} e^{-\frac{1}{2} a|w|^{2}} d w .
$$

In polar coordinates, $w=r \tau, r \geq 0, \tau \in \mathrm{T}$, we expand

$$
(w+\bar{z})^{q} \bar{w}^{q}=\left(r^{2}+\bar{z} r \bar{\tau}\right)^{q}=\sum_{\ell=0}^{q}\left(\begin{array}{l}
q \\
\ell
\end{array}\right)(\bar{z} r \bar{\tau})^{\ell} r^{2(q-\ell)}
$$

and

$$
e^{-\frac{1}{2} a w z}=\sum_{s=0}^{\infty} \frac{1}{s !}\left(-\frac{1}{2} a r z\right)^{s} \tau^{s}
$$

to obtain that the integral in question is equal to

$$
2 \pi e^{-\frac{1}{4} a|z|^{2}} \int_{0}^{\infty} e^{-\frac{1}{2} a r^{2}} r \int_{\mathrm{T}} \sum_{s=0}^{\infty}\left(\frac{1}{s !}\left(-\frac{1}{2} a r z\right)^{s} \tau^{s}\right) \sum_{\ell=0}^{q}\left(\begin{array}{l}
q \\
\ell
\end{array}\right)(\bar{z} r \bar{\tau})^{\ell} r^{2(q-\ell)} d \tau d r .
$$

Using the orthogonality relations we find that the integral over $\mathrm{T}$ is equal to 
$r^{2 q} \sum_{\ell=0}^{q} \frac{1}{\ell !}\left(\begin{array}{c}q \\ \ell\end{array}\right)|z|^{2 \ell}\left(-\frac{1}{2} a\right)^{\ell}$. We don't care about the integral over $r$ (although it is easy to compute), and conclude that the $j^{\text {th }}$ integral is equal to

$$
e^{-\frac{1}{4} a_{k}\left|z_{j}\right|^{2}} \sum_{\ell=0}^{q_{k}^{(j)}} \frac{1}{\ell !}\left(\begin{array}{c}
q_{k}^{(j)} \\
\ell
\end{array}\right)\left|z_{j}\right|^{2 \ell}\left(-\frac{1}{2} a_{k}\right)^{\ell}
$$

up to a constant factor. For the matrix coefficient $\varphi_{k}$ we find the result

$$
\varphi_{k}\left(s, z_{1}, \ldots, z_{n}, t\right)=c_{k}^{\prime} e^{i \lambda_{k} t} e^{-\frac{1}{4} a_{k}|z|^{2}} \prod_{j=1}^{n} \sum_{\ell=0}^{q_{k}^{(j)}} \frac{1}{\ell !}\left(\begin{array}{c}
q_{k}^{(j)} \\
\ell
\end{array}\right)\left|z_{j}\right|^{2 \ell}\left(-\frac{1}{2} a_{k}\right)^{\ell}
$$

with some constant $c_{k}^{\prime}$. Inserting $z=t=0$ yields $c_{k}^{\prime}=1$.

Passing for the last time to a subsequence we may suppose that the $n$ sequences $2 q_{k}^{(j)} a_{k}=2 m_{k}^{(j)} \lambda_{k}$ converge in $[0, \infty]$, i.e., one of cases (1), (2), (3) of Lemma 2.1 occurs for each $j$. Since we know that $\left(\varphi_{k}\right)$ converges uniformly on compacta we conclude from (2.1) that the limit $b_{j}:=\lim _{k \rightarrow \infty}\left(2 \lambda_{k} m_{k}^{(j)}\right)^{\frac{1}{2}}$ is finite for all $j$ and that $\left(\varphi_{k}\right)$ converges to the function

$$
\left(s, z_{1}, \ldots, z_{n}, t\right) \mapsto \prod_{j=1}^{n} J_{0}\left(b_{j}\left|z_{j}\right|\right)
$$

on G. Comparing with the known limit $\varphi$, see (2.8), yields $\left|\gamma_{j}\right|=b_{j}=$ $\lim _{k \rightarrow \infty}\left(2 \lambda_{k} m_{k}^{(j)}\right)^{\frac{1}{2}}$.

This completes the proof for the continuity of $G^{\wedge} \rightarrow \mathfrak{g}_{\text {int }}^{*} / G$. To see the continuity of $\mathfrak{g}_{\mathrm{int}}^{*} / G \rightarrow G^{\wedge}$ it suffices to show that $\pi_{\omega_{k}, \lambda_{k}}$ converges to $\pi_{\omega, \gamma}$ if the criterion of the theorem is fulfilled. Again this task can be reduced to $\omega=1$. To prove the claimed convergence one has to construct unit vectors $\xi$ and $\xi_{k}$ in the spaces of $\pi_{\omega, \lambda}$ and $\pi_{\omega_{k}, \lambda_{k}}$, respectively, such that $\left(\varphi_{k}\right)$ converges to $\varphi$ where $\varphi_{k}$ and $\varphi$ denote the corresponding matrix coefficients. Clearly, one takes the above considered vectors, and proves that $\left(\varphi_{k}\right)$ converges to $\varphi$ by applying Lemma 2.1 directly.

\section{§3. The Primitive Ideal Space of General Diamond Groups}

Using the results of the previous sections we shall now determine the dual topology of general diamond groups $S \ltimes N$. More generally, we also allow that another second countable locally compact abelian group $B$ acts by multiplication with characters on $S \ltimes N$, we assume a continuous homomorphism 


$$
\eta: B \rightarrow S^{\wedge} \text { with dual } \widehat{\eta}: S \rightarrow B^{\wedge}, \widehat{\eta}(s)(b)=\eta(b)(s) .
$$

Such an $\eta$ gives rise to an action $E^{\eta}$ of $B$ on $L^{1}(S \ltimes N)$, namely

$$
\left(E_{b}^{\eta} \varphi\right)(s x)=\varphi(s x) \eta(b)(s)
$$

for $b \in B, s \in S, x \in N$ and $\varphi \in L^{1}(S \ltimes N)$, and we may form the associated covariance algebra $L^{1}\left(B, L^{1}(S \ltimes N), E^{\eta}\right)$ as well as its $C^{*}$-completion $C^{*}\left(B, C^{*}\left(S \ltimes N, E^{\eta}\right)\right.$. The algebra $L^{1}\left(B, L^{1}(S \ltimes N), E^{\eta}\right)$ is isomorphic to $L^{1}\left(S, L^{1}(N) \otimes L^{1}(B), T\right)$, where $\otimes$ means the projective tensor product, i.e., $L^{1}(N) \otimes L^{1}(B)$ is isometrically isomorphic to $L^{1}(N \times B)$, and the action $T$ of $S$ on $L^{1}(N \times B)$ is given by

$$
\left(T_{s} h\right)(x, b)=h\left(\alpha(s)^{-1}(x), b\right) \widehat{\eta}(s)(b)^{-1},
$$

where $\alpha: S \rightarrow U$ is as in (1.4).

The map

$$
\begin{aligned}
& V: L^{1}\left(B, L^{1}(S \ltimes N), E^{\eta}\right) \rightarrow L^{1}\left(S, L^{1}(N \times B), T\right), \\
& (V \psi)(s, x, b)=\eta(b)(s) \psi(b, s, x),
\end{aligned}
$$

is an isometric $*$-isomorphism as it is easily checked. Therefore, also the $C^{*}$ hulls $C^{*}\left(B, C^{*}(S \ltimes N), E^{\eta}\right)$ and $C^{*}\left(S, C^{*}(N) \otimes C^{*}(B), T\right)$ are isomorphic. One step more general we shall investigate $C^{*}$-covariance algebras $C^{*}\left(S, C^{*}(N) \otimes \mathscr{B}, T\right)$ where $\mathscr{B}$ is an arbitrary separable commutative $C^{*}$-algebra, and $S$ acts diagonally on $C^{*}(N) \otimes \mathscr{B}$, like a diamond group on the first factor and arbitrarily on the second, i.e.,

$$
\left(T_{s} h\right)(x)=h\left(\alpha(s)^{-1}(x)\right)^{s^{-1}}
$$

for $s \in S, \quad x \in N$ and an $L^{1}$-function $h: M \rightarrow \mathscr{B}$ where $\mathscr{B} \times S \rightarrow \mathscr{B}$, $(a, s) \mapsto a^{s}$, is any given strongly continuous action of $S$ by isometric $*$-isomorphisms. We just remark that it is not necessary to go to the $C^{*}$-hulls. With some additional effort one can show that all our results in the previous and in the following sections remain true if we stay with $L^{1}$-covariance algebras and if instead of a separable commutative $C^{*}$-algebra $\mathscr{B}$ we consider a separable commutative symmetric regular Banach $*$-algebra $\mathscr{B}$ with a bounded approximate identity. A little more precisely, all the $L^{1}$-versions of the $C^{*}$-algebras we are going to investigate are $*$-regular in the sense of [3], i.e., their spaces of kernels of irreducible involutive representations endowed with the Jacobson topology are homeomorphic to the primitive ideal space of their $C^{*}$-completions. And for the algebra we studied already, namely $L^{1}(U \ltimes N)$, this is equally true because clearly $U \ltimes N$ has a polynomially growing Haar measure, see [3].

Next we write down a collection of irreducible involutive representations 
of $C^{*}\left(S, C^{*}(N) \otimes \mathscr{B}, T\right)$ which exhausts the dual up to weak equivalence. As $Z N$ is central in our algebra or, more rigorously, as point measures at elements in $Z N$ yield central elements in the corresponding adjoint (or multiplier) algebra, again we distinguish between the representations which are trivial on $Z N$ and those which are equal to a non-trivial unitary character $(0, t) \mapsto e^{i \lambda t}$ for $(0, t) \in Z N \subset N=\mathrm{C}^{n} \times \mathrm{R}$. Representations of the first type can be considered as representations of $C^{*}\left(S, C^{*}(N / Z N) \otimes \mathscr{B}, T\right)$. As $C^{*}(N / Z N) \otimes \mathscr{B}$ is commutative the results of $[17,22]$ apply. First we observe that $S$ acts on the structure space $\mathscr{B}^{\wedge}$ of $\mathscr{B}$ by $(s \beta)(b)=\beta\left(b^{s}\right)$. The structure space of $C^{*}(N / Z N)$ can be identified with the Pontrjagin dual $(N / Z N)^{\wedge}$, $\gamma(f)=\int_{N / Z N} f(x) \gamma(x) d x$ for $f \in L^{1}(N / Z N)$ and $\gamma \in(N / Z N)^{\wedge}$, and $S$ acts on $(N / Z N)^{\wedge}$ by $(s \gamma)(x)=\gamma\left(\alpha(s)^{-1}(x)\right)$. Clearly, the structure space of $C^{*}(N / Z N) \otimes \mathscr{B}$ can be identified with $(N / Z N)^{\wedge} \times \mathscr{B}^{\wedge}$, and $S$ acts diagonally on this product.

For $\sigma \in S^{\wedge}, \gamma \in(N / Z N)^{\wedge}$ and $\beta \in \mathscr{B}^{\wedge}$ define a representation $\pi_{\sigma, \gamma, \beta}$ in $L^{2}\left(S /\left(S_{\gamma} \cap S_{\beta}\right)\right)$, where $S_{\gamma} \cap S_{\beta}$ of course denotes the common stabilizer of $\gamma$ and $\beta$ in $S$, by a covariance pair $\pi_{\sigma, \gamma, \beta}^{(1)}, \pi_{\sigma, \gamma, \beta}^{(2)}$. The unitary representation $\pi_{\sigma, \gamma, \beta}^{(1)}$ of $S$ is defined by

$$
\left(\pi_{\sigma, \gamma, \beta}^{(1)}(s) \xi\right)(t)=\sigma(s) \xi\left(s^{-1} t\right)
$$

and the involutive representation $\pi_{\sigma, \gamma, \beta}^{(2)}$ of $C^{*}(N / Z N) \otimes \mathscr{B}$ is defined by

$$
\left(\pi_{\sigma, \gamma, \beta}^{(2)}(f \otimes b) \xi\right)(t)=\beta\left(b^{t}\right)(t \gamma)(f) \xi(t)
$$

for $\xi \in L^{2}\left(S /\left(S_{\gamma} \cap S_{\beta}\right)\right), t \in S /\left(S_{\gamma} \cap S_{\beta}\right)$.

The kernels of those representations exhaust Priv $\left(S, C^{*}(N / Z N) \otimes \mathscr{B}, T\right)$, and one has $\operatorname{ker}_{C^{*}} \pi_{\sigma, \gamma, \beta}=\operatorname{ker}_{C^{*}} \pi_{\sigma^{\prime}, \gamma^{\prime}, \beta^{\prime}}$ if and only if

$$
(S \cdot(\gamma, \beta))^{-}=\left(S \cdot\left(\gamma^{\prime}, \beta^{\prime}\right)\right)^{-} \text {and } \sigma=\sigma^{\prime} \text { on } S_{\gamma} \cap S_{\beta} .
$$

In this way be obtain an open equivalence relation $\sim$ on $S^{\wedge} \times(N / Z N)^{\wedge} \times$ $\mathscr{B}^{\wedge}$, and Priv $\left(S, C^{*}(N / Z N) \otimes \mathscr{B}, T\right)$ is homeomorphic to the quotient space $\left(S^{\wedge} \times(N / Z N)^{\wedge} \times \mathscr{B}^{\wedge}\right) / \sim$.

The representations of the second type lying over a fixed chosen non-zero $\lambda$ can be considered as representations of $C^{*}\left(S, C^{*}(N)_{\lambda} \otimes \mathscr{B}, T\right)$ where $C^{*}(N)_{\lambda}$ is the $C^{*}$-completion of the algebra $L^{1}(N)_{\lambda}$ consisting of all measurable functions $f: N \rightarrow \mathrm{C}$ such that $f\left(z, t^{\prime}+t\right)=f\left(z, t^{\prime}\right) e^{-i \lambda t}$ for all $z \in \mathrm{C}^{n}$, $t, t^{\prime} \in \mathrm{R}$ and

$$
\int_{\mathrm{C}^{n}}|f(z, 1)| d z<\infty .
$$

By means of the representation $\pi_{\lambda}$, compare $(2.3), C^{*}(N)_{\lambda}$ can be identified 
with the algebra $\mathfrak{K}\left(\mathfrak{F}_{\lambda}\right)$ of compact operators on $\mathfrak{F}_{\lambda}$. Under this identification the action of $S$ on $C^{*}(N)_{\lambda}$ transforms into

$$
T_{S}(A)=K_{\lambda}(\alpha(s)) A K_{\lambda}(\alpha(s))^{-1}
$$

for $s \in S, A \in \mathfrak{K}\left(\mathfrak{F}_{\lambda}\right)$, where $K_{\lambda}$ is as in (2.4). Using this group $s \mapsto K_{\lambda}(\alpha(s))$ of unitary operators one can show that $C^{*}\left(S, C^{*}(N)_{\lambda} \otimes \mathscr{B}, T\right)$ is isomorphic to $\mathfrak{K}\left(\mathfrak{F}_{\lambda}\right) \otimes C^{*}(S, \mathscr{B}, T)$. Therefore, the space of primitive ideals in $C^{*}\left(S, C^{*}(N) \otimes \mathscr{B}, T\right)$ lying over $\lambda$ is homeomorphic to $\operatorname{Priv}(S, \mathscr{B}, T)$ which is homeomorphic to $\left(S^{\wedge} \times \mathscr{B}^{\wedge}\right) / \sim$ where

$$
(\sigma, \beta) \sim\left(\sigma^{\prime}, \beta^{\prime}\right) \text { if }(S \beta)^{-}=\left(S \beta^{\prime}\right)^{-} \text {and } \sigma=\sigma^{\prime} \text { on } S_{\beta} .
$$

More explicitly, for each triple $(\sigma, \lambda, \beta) \in S^{\wedge} \times \mathbf{R}^{\times} \times \mathscr{B}^{\wedge}$ we define a representation $\pi_{\sigma, \lambda, \beta}$ of $C^{*}\left(S, C^{*}(N) \otimes \mathscr{B}, T\right)$ in $L^{2}\left(S / S_{\beta}, \mathfrak{F}_{\lambda}\right)$ by a covariance pair $\pi_{\sigma, \lambda, \beta}^{(1)}, \pi_{\sigma, \lambda, \beta}^{(2)}$ :

$$
\begin{aligned}
\left(\pi_{\sigma, \lambda, \beta}^{(1)}(s) \xi\right)(t) & =\sigma(s) K_{\lambda}(\alpha(s)) \xi\left(s^{-1} t\right), \\
\left(\pi_{\sigma, \lambda, \beta}^{(2)}(f \otimes b) \xi\right)(t) & =\beta\left(b^{t}\right) \pi_{\lambda}(f) \xi(t)
\end{aligned}
$$

for $\xi \in L^{2}\left(S / S_{\beta}, \mathfrak{F}_{\lambda}\right), t \in S / S_{\beta}, s \in S, f \in C^{*}(N)$ and $b \in \mathscr{B}$.

In that way we get all irreducible representation of the second type up to weak equivalence, and this parametrization is also topologically alright:

Remark 3.9. The map $(\sigma, \lambda, \beta) \mapsto \operatorname{ker}_{C^{*}} \pi_{\sigma, \lambda, \beta}$ from $S^{\wedge} \times \mathrm{R}^{\times} \times \mathscr{B}^{\wedge}$ into the open subset $\operatorname{Priv}\left(S, C^{*}(N) \otimes \mathscr{B}, T\right) \backslash \operatorname{Priv}\left(S, C^{*}(N / Z N) \otimes \mathscr{B}, T\right) \quad$ of Priv $\left(S, C^{*}(N) \otimes \mathscr{B}, T\right)$ is surjective, and one has $\operatorname{ker}_{C^{*}} \pi_{\sigma, \lambda, \beta}=\operatorname{ker}_{C^{*}} \pi_{\sigma^{\prime}, \lambda^{\prime}, \beta^{\prime}}$ if and only if $\lambda=\lambda^{\prime}$, and $(\sigma, \beta)$ and $\left(\sigma^{\prime}, \beta^{\prime}\right)$ are equivalent in the sense of (3.7). This way one obtains an (open) equivalence relation, also denoted by $\sim$, on $S^{\wedge} \times \mathrm{R}^{\times} \times \mathscr{B}^{\wedge}$, and

$$
\operatorname{Priv}\left(S, C^{*}(N) \otimes \mathscr{B}, T\right) \backslash \operatorname{Priv}\left(S, C^{*}(N / Z N) \otimes \mathscr{B}, T\right)
$$

is homeomorphic to $\left(S^{\wedge} \times \mathrm{R}^{\times} \times \mathscr{B}^{\wedge}\right) / \sim$.

Proof. Most things are clear in view of the former considerations. The only new item is the claimed homeomorphy. As in the proof of Theorem 2.6 we use that the group $\mathbf{R}_{+}$of positive real numbers acts by dilations on $C^{*}\left(S, C^{*}(N) \otimes \mathscr{B}, T\right)$. For $r \in \mathrm{R}_{+}$and an $L^{1}$-function $f: S \times N \rightarrow \mathscr{B}$ define $f^{r}: S \times N \rightarrow \mathscr{B}$ by

$$
f^{r}(s, z, t)=r^{2 n+2} f\left(s, r z, r^{2} t\right) .
$$

This action extends to a strongly continuous action of $\mathbf{R}_{+}$by $*$-automorphisms on $C^{*}\left(S, C^{*}(N) \otimes \mathscr{B}, T\right)$. Suppose that a sequence of representations $\pi_{\sigma_{k}, \lambda_{k}, \beta_{k}}, k \in \mathrm{N}$, converges to $\pi_{\sigma, \lambda, \beta}$. Then clearly $\left(\lambda_{k}\right)$ converges to $\lambda$, whence 
$r_{k}:=\left(\frac{\lambda_{k}}{\lambda}\right)^{\frac{1}{2}}$ converges to 1 . Therefore, the modified sequence $r_{k} \cdot \pi_{\sigma_{k}, \lambda_{k}, \beta_{k}}$, defined by $\left(r_{k} \cdot \pi_{\sigma_{k}, \lambda_{k}, \beta}\right)(f)=\left(\pi_{\sigma_{k}, \lambda_{k}, \beta_{k}}\right)\left(f^{r_{k}}\right)$ for $f \in C^{*}\left(S, C^{*}(N) \otimes \mathscr{B}, T\right)$, also converges to $\pi_{\sigma, \lambda, \beta}$. It is easy to check that $r_{k} \cdot \pi_{\sigma_{k}, \lambda_{k}, \beta_{k}}$ is unitarily equivalent to $\pi_{\sigma_{k}, \lambda, \beta_{k}}$. Thus we are reduced to $\lambda_{k}=\lambda$. But in this case the above consideration on the isomorphy of $C^{*}\left(S, C^{*}(N)_{\lambda} \otimes \mathscr{B}, T\right)$ with $\mathfrak{K}\left(\mathfrak{F}_{\lambda}\right) \otimes$ $C^{*}(S, \mathscr{B}, T)$ applies, and shows that the equivalence classes through $\left(\sigma_{k}, \beta_{k}\right)$ converge to the equivalence class through $(\sigma, \beta)$. This proves one part of the homeomorphy, the other one can be done along the same line.

Just we remark that there is even a global picture: The ideal $\mathscr{I}:=\cap P$, $P \in \operatorname{Priv}\left(S, C^{*}(N / Z N) \otimes \mathscr{B}, T\right)$, in $C^{*}\left(S, C^{*}(N) \otimes \mathscr{B}, T\right)$ is indeed isomorphic to $C^{*}(S, \mathscr{B}, T) \otimes \mathscr{C}_{\infty}\left(\mathrm{R}^{\times}, \mathfrak{K}\right)$ where $\mathfrak{K}$ denotes the algebra of compact operators on a separable Hilbert space.

In summary, we know the topology of the closed subspace $\operatorname{Priv}\left(S, C^{*}(N / Z N) \otimes \mathscr{B}, T\right)$ of Priv $\left(S, C^{*}(N) \otimes \mathscr{B}, T\right)$ and of its open complement. To know the whole topology we need a criterion to decide which sequences in the open set converge to points in the closed subset. To this end, we introduce an auxiliary $C^{*}$-algebra with the same spectrum, which will also lead to another parametrization.

We let $U \times S$ act on $C^{*}(N) \otimes \mathscr{B}$ by

$$
\left(R_{u, s}^{\prime} f\right)(x)=f\left(\zeta\left(u^{-1} \alpha(s)^{-1}\right)(x)\right)^{s^{-1}}
$$

for $L^{1}$-functions $f: N \rightarrow \mathscr{B}$. On the resulting covariance algebra $C^{*}(U \times S$, $\left.C^{*}(N) \otimes \mathscr{B}, R^{\prime}\right)$ we let the Pontrjagin dual $U^{\wedge}$ act by

$$
\left(E_{\omega}^{\prime} \varphi\right)(u, s)=\omega(u) \varphi(u, s)
$$

for $L^{1}$-functions $\varphi: U \times S \rightarrow C^{*}(N) \otimes \mathscr{B}$. By Takai duality, see [20], the covariance algebra

$$
C^{*}\left(U^{\wedge}, C^{*}\left(U \times S, C^{*}(N) \otimes \mathscr{B}, R^{\prime}\right), E^{\prime}\right)
$$

is isomorphic to $C^{*}\left(S, C^{*}(N) \otimes \mathscr{B}, T\right) \otimes \mathfrak{K}\left(L^{2}(U)\right)$. The algebra $C^{*}(U \times$ $\left.S, C^{*}(N) \otimes \mathscr{B}, R^{\prime}\right)$ is isomorphic to $C^{*}\left(S \times U, C^{*}(N) \otimes \mathscr{B}, R\right)$ where the action $R$ is defined by

$$
R_{s, u}=R_{u \alpha(s)^{-1}, s}^{\prime}, \text { i.e. }\left(R_{s, u} f\right)(x)=f\left(\zeta\left(u^{-1}\right)(x)\right)^{s^{-1}}
$$

for $\quad L^{1}$-functions $\quad f: N \rightarrow \mathscr{B} ; \quad$ an isomorphism $A: C^{*}\left(U \times S, C^{*}(N) \otimes\right.$ $\left.b, R^{\prime}\right) \rightarrow C^{*}\left(S \times U, C^{*}(N) \otimes \mathscr{B}, R\right)$ is given by

$$
(A \varphi)(s, u)=\varphi\left(u \alpha(s)^{-1}, s\right) .
$$

Therefore, the algebra $C^{*}\left(U^{\wedge}, C^{*}\left(U \times S, C^{*}(N) \otimes \mathscr{B}, R^{\prime}\right), E^{\prime}\right)$ is isomorphic 
to $C^{*}\left(U^{\wedge}, C^{*}\left(S \times U, C^{*}(N) \otimes \mathscr{B}, R\right), E\right)$ if the action $E^{\prime}$ is transformed accordingly into an action $E$ of $U^{\wedge}$, namely

$$
\left(E_{\omega} \varphi\right)(s, u)=\omega\left(u \alpha(s)^{-1}\right) \varphi(s, u)
$$

for $\omega \in U^{\wedge}$ and $L^{1}$-functions $\varphi: S \times U \rightarrow C^{*}(N) \otimes \mathscr{B}$. The advantage of this simple transformation of variables is that $C^{*}\left(S \times U, C^{*}(N) \otimes \mathscr{B}, R\right)$ is isomorphic to the tensor product of $C^{*}(U \ltimes N)$ with $C^{*}(S, \mathscr{B}, T)$, whose spectra we know. Clearly we have to study how the representations of $C^{*}\left(S, C^{*}(N) \otimes \mathscr{B}, T\right)$ are transformed. Let $\tau$ be a representation of the latter algebra given by a covariance pair $\tau^{(1)}, \tau^{(2)}$ in some Hilbert space $\mathfrak{H}$. Takai duality yields a corresponding representation $\mu^{\prime}$ of $C^{*}\left(U^{\wedge}, C^{*}(U \times\right.$ $\left.\left.S, C^{*}(N) \otimes \mathscr{B}, R^{\prime}\right), E^{\prime}\right)$ in $L^{2}(U, \mathfrak{H})$ given by triple $\mu^{\prime(0)}, \mu^{\prime(1)}, \mu^{\prime(2)}$ where $\mu^{\prime(0)}$ and $\mu^{\prime(1)}$ are unitary representations of $U^{\wedge}$ and $U \times S$, respectively, and $\mu^{\prime(2)}$ is an involutive representation of $C^{*}(N) \otimes \mathscr{B}$, namely

$$
\begin{aligned}
\left(\mu^{\prime(0)}(\omega) \xi\right)(v) & =\omega(v) \xi(v), \\
\left(\mu^{\prime(1)}(u, s) \xi\right)(v) & =\tau^{(1)}(s)\left(\xi\left(u^{-1} v\right)\right), \\
\left(\mu^{\prime(2)}(f \otimes b) \xi\right)(v) & =\tau^{(2)}\left(f^{v} \otimes b\right)(\xi(v))
\end{aligned}
$$

for $v \in U, \xi \in L^{2}(U, \mathfrak{H})$, where $f^{v}$ is defined for $f \in L^{1}(N)$ by $f^{v}(x)=$ $f(\zeta(v)(x))$. The covariance pair $\mu^{\prime(1)}, \mu^{\prime(2)}$ yields a representation of $C^{*}\left(U \times S, C^{*}(N) \otimes \mathscr{B}, R^{\prime}\right)$ which together with $\mu^{\prime(0)}$ forms a covariance pair for the whole algebra; the latter pair gives $\mu^{\prime}$. Transforming along $A$ leads to a representation $\mu$ of $C^{*}\left(U^{\wedge}, C^{*}\left(S \times U, C^{*}(N) \otimes \mathscr{B}, R\right), E\right)$ which similarily is given by a triple $\mu^{(0)}, \mu^{(1)}, \mu^{(2)}$, namely

$$
\begin{aligned}
& \mu^{(0)}=\mu^{(0)}, \mu^{(2)}=\mu^{\prime(2)} \text { and } \\
& \mu^{(1)}(s, u)=\mu^{\prime(1)}\left(u \alpha(s)^{-1}, s\right), \text { i.e. } \\
& \left(\mu^{(1)}(s, u) \xi\right)(v)=\tau^{(1)}(s)\left(\xi\left(u^{-1} \alpha(s) v\right)\right)
\end{aligned}
$$

for $\xi \in L^{2}(U, \mathfrak{H})$.

Knowing the set $\operatorname{Priv}\left(S, C^{*}(N) \otimes \mathscr{B}, T\right)$ we also know the set $\operatorname{Priv}\left(U^{\wedge}, C^{*}\left(S \times U, C^{*}(N) \otimes \mathscr{B}, R\right), E\right)$, but there is a more natural and useful parametrization of the latter set which we describe next. Again we distinguish the irreducible representations/primitive ideals according to their behaviour on $Z N$. The algebra $C^{*}\left(U^{\wedge}, C^{*}\left(S \times U, C^{*}(N / Z N) \otimes \mathscr{B}, R\right), E\right)$ is of the type studied in [17, Proposition 3.5]. Its primitive ideal space is parametrized by $U \times S^{\wedge} \times U^{\wedge} \times(N / Z N)^{\wedge} \times \mathscr{B}^{\wedge}$ : For $\left(u_{o}, \sigma_{0}, \omega_{0}, \gamma_{0}, \beta_{0}\right)$ in this product define a representation $\chi=\chi_{u_{0}, \sigma_{0}, \omega_{0}, \gamma_{0}, \beta_{0}}$ as usual by a triple $\chi^{(0)}, \chi^{(1)}, \chi^{(2)}$ in the space $L^{2}((S \times U) / F)$ where the subgroup $F$ consists of 
all pairs $(s, \alpha(s))$ with $s \in S_{\beta_{0}}$ and $\alpha(s) \in U_{\gamma_{0}}$, i.e., $s$ is in the stabilizer of $\left(\gamma_{0}, \beta_{0}\right)$ with respect to the original action of $S$ on $\left(C^{*}(N) \otimes \mathscr{B}\right)^{\wedge}$, namely

$$
\begin{aligned}
\left(\chi^{(0)}(\omega) \xi\right)\left(s^{\prime}, u^{\prime}\right) & =\omega\left(u_{0} u^{\prime} \alpha\left(s^{\prime}\right)^{-1}\right) \xi\left(s^{\prime}, u^{\prime}\right) \\
\left(\chi^{(1)}(s, u) \xi\right)\left(s^{\prime}, u^{\prime}\right) & =\sigma_{0}(s) \omega_{0}(u) \xi\left(s^{-1} s^{\prime}, u^{-1} u^{\prime}\right) \\
\left(\chi^{(2)}(f \otimes b) \xi\right)\left(s^{\prime}, u^{\prime}\right) & =\gamma_{0}\left(f^{u^{\prime}}\right) \beta_{0}\left(b^{s^{\prime}}\right) \xi\left(s^{\prime}, u^{\prime}\right)
\end{aligned}
$$

for $\xi \in L^{2}((S \times U) / F), \quad\left(s^{\prime}, u^{\prime}\right) \in(S \times U) / F, \omega \in U^{\wedge}, \quad(s, u) \in S \times U$ and $f \otimes b \in C^{*}(N) \otimes \mathscr{B}$.

(3.18) The map $(u, \sigma, \omega, \gamma, \beta) \mapsto \operatorname{ker}_{C^{*}} \chi_{u, \sigma, \omega, \gamma, \beta}$ from the product space $U \times S^{\wedge} \times U^{\wedge} \times(N / Z N)^{\wedge} \times \mathscr{B}^{\wedge} \quad$ into $\quad$ Priv $\left(U^{\wedge}, C^{*}\left(S \times U, C^{*}(N / Z N) \otimes\right.\right.$ $\mathscr{B}, R), E)$ is continuous, open and onto.

The equation

$$
\operatorname{ker}_{C^{*}} \chi_{u, \sigma, \omega, \gamma, \beta}=\operatorname{ker}_{C^{*}} \chi_{u_{0}, \sigma_{o}, \omega_{0}, \gamma_{o}, \beta_{o}}
$$

defines an (open) equivalence relation $\sim$ on $U \times S^{\wedge} \times U^{\wedge} \times(N / Z N)^{\wedge} \times \mathscr{B}^{\wedge}$ which by [17, Proposition 3.5] can be described as follows. Let $\left(s^{\prime}, u^{\prime}\right) \in S \times U$ act on $(u, \gamma, \beta) \in U \times(N / Z N)^{\wedge} \times \mathscr{B}^{\wedge}$ by

$$
\left(s^{\prime}, u^{\prime}\right) \cdot(u, \gamma, \beta)=\left(u^{\prime} \alpha\left(s^{\prime}\right)^{-1} u, u^{\prime} \cdot \gamma, s^{\prime} \cdot \beta\right)
$$

which means that the stabilizer of $(u, \gamma, \beta) \in U \times(N / Z N)^{\wedge} \times \mathscr{B}^{\wedge}$ in $S \times U$ consists of all $\left(s^{\prime}, \alpha\left(s^{\prime}\right)\right) \in S \times U$ with $s^{\prime} \in S_{\beta}$ and $\alpha\left(s^{\prime}\right) \in U_{\gamma}$. Then

$$
\left(u_{0}, \sigma_{0}, \omega_{0}, \gamma_{0}, \beta_{0}\right) \sim(u, \sigma, \omega, \gamma, \beta)
$$

if and only if the closures of the $(S \times U)$-orbits through $(u, \gamma, \beta)$ and $\left(u_{0}, \gamma_{0}, \beta_{0}\right)$ coincide and if $\left(\sigma_{0}, \omega_{0}\right)=(\sigma, \omega)$ on the stabilizer of $(u, \gamma, \beta) \in$ $U \times(N / Z N)^{\wedge} \times \mathscr{B}^{\wedge}$ in $S \times U$, i.e., $\sigma_{0}\left(s^{\prime}\right) \omega_{0}\left(\alpha\left(s^{\prime}\right)\right)=\sigma\left(s^{\prime}\right) \omega\left(\alpha\left(s^{\prime}\right)\right)$ for all $s^{\prime} \in S_{\beta}$ with $\alpha\left(s^{\prime}\right) \in U_{\gamma}$.

Using the compactness of $U$ it is not hard to see that the condition on the closures of the $(S \times U)$-orbits can be rephrased by saying that the $S$-quasiorbits through $\left(\left(u_{0} u^{-1}\right) \cdot \gamma, \beta\right)$ and $\left(\gamma_{0}, \beta_{0}\right)$ for the original $S$-action coincide, i.e.,

$$
\left(S^{\text {ori }} \cdot\left(\left(u_{0} u^{-1}\right) \cdot \gamma, \beta\right)\right)^{-}=\left(S^{\text {ori }} \cdot\left(\gamma_{0}, \beta_{0}\right)\right)^{-}
$$

where $s \cdot(\gamma, \beta)=\left(\gamma^{\prime}, s \cdot \beta\right)$ with $\gamma^{\prime}(x)=\gamma\left(\alpha(s)^{-1}(x)\right)$ for $x \in N$.

From the structure of the action $E$ and the known representation theory of $C^{*}\left(S \times U, C^{*}(N) \otimes \mathscr{B}, R\right) \cong C^{*}(S, \mathscr{B}, T) \otimes C^{*}(U \ltimes N)$ it easily follows that the primitive ideals of $C^{*}\left(U^{\wedge}, C^{*}\left(S \times U, C^{*}(N) \otimes \mathscr{B}, R\right), E\right)$ in general position are induced from $C^{*}\left(S \times U, C^{*}(N) \otimes \mathscr{B}, R\right)$. We parametrize those ideals by $\left(u_{0}, \sigma_{0}, \omega_{0}, \lambda_{0}, \beta_{0}\right) \in U \times S^{\wedge} \times U^{\wedge} \times \mathbf{R}^{\times} \times \mathscr{B}^{\wedge}$. The parameter $u_{0}$ is 
superfluous, but included for almost evident reasons. Again the representation $\chi=\chi_{u_{0}, \sigma_{0}, \omega_{0}, \lambda_{0} \beta_{0}}$ is given by a triple $\chi^{(0)}, \chi^{(1)}, \chi^{(2)}$. It acts on the space $L^{2}\left(U^{\wedge} \times\left(S / S_{\beta_{0}}\right), \mathfrak{F}_{\lambda_{0}}\right)$ by:

$$
\begin{aligned}
\left(\chi^{(0)}(\omega) \xi\right)\left(\omega^{\prime}, s^{\prime}\right) & =\xi\left(\omega^{-1} \omega^{\prime}, s^{\prime}\right), \\
\left(\chi^{(1)}(s, u) \xi\right)\left(\omega^{\prime}, s^{\prime}\right) & =\omega^{\prime}\left(u^{-1} \alpha(s)\right) \sigma_{0}(s) \omega_{0}(u) K_{\lambda_{0}}(u)\left(\xi\left(\omega^{\prime}, s^{-1} s^{\prime}\right)\right), \\
\left(\chi^{(2)}(f \otimes b) \xi\right)\left(\omega^{\prime}, s^{\prime}\right) & =\beta_{0}\left(b^{s^{\prime}}\right) \pi_{\lambda_{0}}(f)\left(\xi\left(\omega^{\prime}, s^{\prime}\right)\right)
\end{aligned}
$$

for $\xi \in L^{2}\left(U^{\wedge} \times\left(S / S_{\beta_{0}}\right), \mathfrak{F}_{\lambda_{0}}\right),\left(\omega^{\prime}, s^{\prime}\right) \in U^{\wedge} \times\left(S / S_{\beta_{0}}\right)$ where $\pi_{\lambda_{0}}$ and $K_{\lambda_{0}}$ are as in $(2.3 / 4)$.

For the (open) equivalence relation $\sim$ on $U \times S^{\wedge} \times U^{\wedge} \times \mathrm{R}^{\times} \times \mathscr{B}^{\wedge}$ defined by

$$
\operatorname{ker}_{C^{*}} \chi_{u, \sigma, \omega, \lambda, \beta}=\operatorname{ker}_{C^{*}} \chi_{u_{0}, \sigma_{0}, \omega_{0}, \lambda_{0}, \beta_{0}}
$$

one obtains

$$
(u, \sigma, \omega, \lambda, \beta) \sim\left(u_{0}, \sigma_{0}, \omega_{0}, \lambda_{0}, \beta_{0}\right)
$$

if and only if $\lambda=\lambda_{0},(S \beta)^{-}=\left(S \beta_{0}\right)^{-}$and $\left.\widehat{\alpha}\left(\omega_{0}\right) \sigma_{0}\right|_{S_{\beta}}=\left.\widehat{\alpha}(\omega) \sigma\right|_{S_{\beta}}$ where $\widehat{\alpha}: U^{\wedge} \rightarrow S^{\wedge}$ denotes the dual homomorphism, $\widehat{\alpha}(\omega)(s)=\omega(\alpha(s))$.

In $(3.15 / 16)$ we described the general procedure to assign to representations of $C^{*}\left(S, C^{*}(N) \otimes \mathscr{B}, T\right)$ such of $C^{*}\left(U^{\wedge}, C^{*}\left(S \times U, C^{*}(N) \otimes \mathscr{B}, R\right), E\right)$. Applying this procedure to $\pi_{\sigma, \gamma, \beta}$ or $\pi_{\sigma, \lambda, \beta}$ gives some of the $\chi$ 's (up to unitary equivalence). This is explained next in somewhat more detail. To the representation $\pi_{\sigma, \gamma, \beta}$ in $L^{2}\left(S /\left(S_{\gamma} \cap S_{\beta}\right)\right)$ corresponds the triple $\mu^{(0)}, \mu^{(1)}, \mu^{(2)}$ in the space $L^{2}\left(U \times\left(S / S_{\gamma} \cap S_{\beta}\right)\right)$ :

$$
\begin{aligned}
\left(\mu^{(0)}(\omega) \xi\right)\left(u^{\prime}, s^{\prime}\right) & =\omega\left(u^{\prime}\right) \xi\left(u^{\prime}, s^{\prime}\right) \\
\left(\mu^{(1)}(s, u) \xi\right)\left(u^{\prime}, s^{\prime}\right) & =\sigma(s) \xi\left(u^{-1} \alpha(s) u^{\prime}, s^{-1} s^{\prime}\right) \\
\left(\mu^{(2)}(f \otimes b) \xi\right)\left(u^{\prime}, s^{\prime}\right) & =\beta\left(b^{s^{\prime}}\right)\left(\left(\alpha\left(s^{\prime}\right) u^{\prime}\right) \cdot \gamma\right)(f) \xi\left(u^{\prime}, s^{\prime}\right)
\end{aligned}
$$

for $\xi \in L^{2}\left(U \times\left(S / S_{\gamma} \cap S_{\beta}\right)\right), u^{\prime} \in U, s^{\prime} \in S / S_{\gamma} \cap S_{\beta}$.

(3.25) The representation $\mu$ corresponding to $\mu^{(0)}, \mu^{(1)}, \mu^{(2)}$ is unitarily equivalent to $\chi_{1, \sigma, 1, \gamma, \beta}$ in $L^{2}((S \times U) / F)$ where $F=\left\{(s, \alpha(s)) \mid s \in S_{\beta}, \alpha(s) \in\right.$ $\left.U_{\gamma}\right\} \leq S \times U$.

It is easy to verify that the operator

$$
Q: L^{2}((S \times U) / F) \rightarrow L^{2}\left(U \times\left(S / S_{\gamma} \cap S_{\beta}\right)\right)
$$

defined by $(Q \xi)(u, s)=\xi(s, \alpha(s) u)$ intertwines the triples $\mu^{(0)}, \mu^{(1)}, \mu^{(2)}$ and $\chi_{1, \sigma, 1, \gamma, \beta}^{(0)}, \chi_{1, \sigma, 1, \gamma, \beta}^{(1)}, \chi_{1, \sigma, 1, \gamma, \beta}^{(2)}$. 
To the representation $\pi_{\sigma, \lambda, \beta}$ in $L^{2}\left(S / S_{\beta}, \mathfrak{F}_{\lambda}\right)$ corresponds the triple $\mu^{(0)}, \mu^{(1)}, \mu^{(2)}$ in the space $L^{2}\left(U \times\left(S / S_{\beta}\right), \mathfrak{F}_{\lambda}\right)$ :

$$
\begin{aligned}
\left(\mu^{(0)}(\omega) \xi\right)\left(u^{\prime}, s^{\prime}\right) & =\omega\left(u^{\prime}\right) \xi\left(u^{\prime}, s^{\prime}\right) \\
\left(\mu^{(1)}(s, u) \xi\right)\left(u^{\prime}, s^{\prime}\right) & =\sigma(s) K_{\lambda}(\alpha(s)) \xi\left(u^{-1} \alpha(s) u^{\prime}, s^{-1} s^{\prime}\right) \\
\left(\mu^{(2)}(f \otimes b) \xi\right)\left(u^{\prime}, s^{\prime}\right) & =\beta\left(b^{s^{\prime}}\right) \pi_{\lambda}\left(f^{u^{\prime}}\right) \xi\left(u^{\prime}, s^{\prime}\right)
\end{aligned}
$$

for $\xi \in L^{2}\left(U \times\left(S / S_{\beta}\right), \mathfrak{F}_{\lambda}\right), u^{\prime} \in U, s^{\prime} \in S / S_{\beta}$.

(3.28) The representation $\mu$ corresponding to $\mu^{(0)}, \mu^{(1)}, \mu^{(2)}$ is unitarily equivalent to $\chi_{1, \sigma, 1, \lambda, \beta}$ in $L^{2}\left(U^{\wedge} \times\left(S / S_{\beta}\right), \mathfrak{F}_{\lambda}\right)$.

Using $K_{\lambda}(u) \pi_{\lambda}\left(f^{u}\right)=\pi_{\lambda}(f) K_{\lambda}(u)$ for $u \in U, f \in C^{*}(N)$ it is not hard to see that the operator

$$
Q: L^{2}\left(U \times\left(S / S_{\beta}\right), \mathfrak{F}_{\lambda}\right) \rightarrow L^{2}\left(U^{\wedge} \times\left(S / S_{\beta}\right), \mathfrak{F}_{\lambda}\right)
$$

defined by $(Q \xi)(\omega, s)=\int_{U} \omega(u)^{-1} K_{\lambda}(u) \xi(u, s) d u$, i.e, essentially the $L^{2}$-Fourier transform, provides us with a unitary equivalence.

On the level of parameters we find the corresponding picture.

(3.30) The map $I: S^{\wedge} \times(N / Z N)^{\wedge} \times \mathscr{B}^{\wedge} \rightarrow U \times S^{\wedge} \times U^{\wedge} \times(N / Z N)^{\wedge} \times$ $\mathscr{B}^{\wedge}$ defined by $I(\sigma, \gamma, \beta)=(1, \sigma, 1, \gamma, \beta)$ induces a homeomorphism from $\left(S^{\wedge} \times(N / Z N)^{\wedge} \times \mathscr{B}^{\wedge} / \sim\right.$ onto $\left(U \times S^{\wedge} \times U^{\wedge} \times(N / Z N)^{\wedge} \times \mathscr{B}^{\wedge}\right) / \sim$, and the map $I: S^{\wedge} \times \mathrm{R}^{\times} \times \mathscr{B}^{\wedge} \rightarrow U \times S^{\wedge} \times U^{\wedge} \times \mathrm{R}^{\times} \times \mathscr{B}^{\wedge}$ defined $I(\sigma, \lambda, \beta)=$ $(1, \sigma, 1, \lambda, \beta)$ induces a homeomorphism from $\left(S^{\wedge} \times \mathbf{R}^{\times} \times \mathscr{B}^{\wedge}\right) / \sim$ onto $\left(U \times S^{\wedge} \times U^{\wedge} \times \mathrm{R}^{\times} \times \mathscr{B}^{\wedge}\right) / \sim$; for the various equivalence relations compare $(3.6 / 9 / 20 / 23)$.

This should be the case in view of Takai duality, and it can be verified directly. From this fact we conclude:

(3.31) The map $(u, \sigma, \omega, \lambda, \beta) \mapsto \operatorname{ker}_{C^{*}} \chi_{u, \sigma, \omega, \lambda \beta}$ from the topological product $U \times S^{\wedge} \times U^{\wedge} \times \mathrm{R}^{\times} \times \mathscr{B}^{\wedge}$ onto the complement of Priv $\left(U^{\wedge}, C^{*}(S \times\right.$ $\left.\left.U, C^{*}(N / Z N) \otimes \mathscr{B}, R\right), E\right)$ in Priv $\left(U^{\wedge}, C^{*}\left(S \times U, C^{*}(N) \otimes \mathscr{B}, R\right), E\right)$ is open and continuous.

What we have achieved is an evidently more complicated picture of Priv $\left(S, C^{*}(N) \otimes \mathscr{B}, T\right)$. But the pay is twofold. First the primitive ideals in general position are induced from an algebra with known spectrum. Secondly the two pieces $U \times S^{\wedge} \times U^{\wedge} \times(N / Z N)^{\wedge} \times \mathscr{B}^{\wedge}$ and $U \times S^{\wedge} \times U^{\wedge} \times$ $\mathrm{R}^{\times} \times \mathscr{B}^{\wedge}$ can be glued together as the topological product $U \times S^{\wedge} \times \mathfrak{Y} \times \mathscr{B}^{\wedge}$ where $\mathfrak{Y}=U^{\wedge} \times \mathbf{R}^{\times} \cup U^{\wedge} \times(N / Z N)^{\wedge}$ is equipped with the topology given in front of (1.21).

TheOREM 3.32. The map $\Phi^{\prime}$ from $\mathfrak{Q}^{\prime}:=U \times S^{\wedge} \times \mathfrak{Y} \times \mathscr{B}^{\wedge}$ into Priv $\left(U^{\wedge}\right.$, $\left.C^{*}\left(S \times U, C^{*}(N) \otimes \mathscr{B}, R\right), E\right)$ defined on the pieces $U \times S^{\wedge} \times U^{\wedge} \times \mathrm{R}^{\times} \times \mathscr{B}^{\wedge}$ and $U \times S^{\wedge} \times U^{\wedge} \times(N / Z N)^{\wedge} \times \mathscr{B}^{\wedge}$ by $(3.17 / 18 / 22)$ is continuous, open and 
onto. Therefore, $\Phi^{\prime}$ induces a homeomorphism from $\left(U \times S^{\wedge} \times \mathfrak{Y} \times \mathscr{B}^{\wedge}\right) / \sim$ onto $\operatorname{Priv}\left(U^{\wedge}, C^{*}\left(S \times U, C^{*}(N) \otimes \mathscr{B}, R\right), E\right)$ where the equivalence relation $\sim$ on the two pieces is given by (3.20/23).

PRoof. In view of $(3.18 / 31)$ we only have to show that the parametrizations on the two pieces fit together topologically. Let $q_{k}=\left(u_{k}, \sigma_{k}, \omega_{k}, \lambda_{k}, \beta_{k}\right)$ be any sequence in $U \times S^{\wedge} \times U^{\wedge} \times \mathrm{R}^{\times} \times \mathscr{B}^{\wedge}$, and denote by $\chi_{k}=$ $\chi_{u_{k}, \sigma_{k}, \omega_{k}, \lambda_{k}, \beta_{k}}$ the corresponding representation. Let $q=(u, \sigma, \omega, \gamma, \beta)$ be any point in $U \times S^{\wedge} \times U^{\wedge} \times(N / Z N)^{\wedge} \times \mathscr{B}^{\wedge}$, and denote by $\chi=\chi_{u, \sigma, \omega, \gamma, \beta}$ the corresponding representation. We have to prove that $\left(\chi_{k}\right)$ converges to $\chi$ if $\left(q_{k}\right)$ converges to $q$, and that if $\left(\chi_{k}\right)$ converges to $\chi$ there exists a sequence $\left(q_{k}^{\prime}\right)$ such that $q_{k}^{\prime} \sim q_{k}$ and $\left(q_{k}^{\prime}\right)$ converges to $q$ in $\mathfrak{Q}^{\prime}$. The representations $\chi_{k}$ and $\chi$ yield $U^{\wedge}$-quasi-orbits $\Omega_{k}$ and $\Omega$ in the primitive ideal space of $C^{*}\left(S \times U, C^{*}(N) \otimes \mathscr{B}, R\right) \cong C^{*}(U \ltimes N) \otimes C^{*}(S, \mathscr{B}, T)$. This primitive ideal space is parametrized in the obvious way by $\mathfrak{Y} \times S^{\wedge} \times \mathscr{B}^{\wedge}$, and by Theorem 2.6 and [17, Theorem 3.3] this parametrization is continuous and open. In terms of these parameters one has

$$
\Omega_{k}=\left\{\left(\omega_{k}^{\prime}, \lambda_{k}, \sigma_{k}^{\prime}, \beta_{k}^{\prime}\right)\left|\left(S \beta_{k}^{\prime}\right)^{-}=\left(S \beta_{k}\right)^{-}, \widehat{\alpha}\left(\omega_{k}^{\prime}\right) \sigma_{k}^{\prime}\right| S_{\beta_{k}}=\widehat{\alpha}\left(\omega_{k}\right) \sigma_{k} \mid S_{\beta_{k}}\right\},
$$

and

$\Omega=\left\{\left(\omega^{\prime}, \gamma^{\prime}, \sigma^{\prime}, \beta^{\prime}\right) \mid \widehat{\alpha}\left(\omega^{\prime}\right) \sigma^{\prime}=\widehat{\alpha}(\omega) \sigma\right.$ on $\left.S_{\gamma} \cap S_{\beta}, U_{\gamma^{\prime}}=U_{\gamma},\left(S \beta^{\prime}\right)^{-}=(S \beta)^{-}\right\} ;$

for the second equation compare [17, Proposition 3.5]. Since the $\chi_{k}$ are induced from $C^{*}\left(S \times U, C^{*}(N) \otimes \mathscr{B}, R\right)$ and since $U^{\wedge}$ is amenable as an abelian group (the amenability is needed to ensure that restricting an ideal and inducing it again leads to an ideal contained in the original one) the sequence $\left(\chi_{k}\right)$ converges to $\chi$ (and, of course, to all the other points lying over $\Omega$ at the least) if and only if $\left(\Omega_{k}\right)$ converges to $\Omega$ in the space of $U^{\wedge}$-quasi-orbits in Priv $\left(S \times U, C^{*}(N) \otimes \mathscr{B}, R\right)$, which by Lemma 1.9 is equivalent to the existence of $\left(\omega_{k}^{\prime}, \lambda_{k}, \sigma_{k}^{\prime}, \beta_{k}^{\prime}\right) \in \Omega_{k}$ such that the sequence $\left(\omega_{k}^{\prime}, \lambda_{k}, \sigma_{k}^{\prime}, \beta_{k}^{\prime}\right)$ converges to $(\omega, \gamma, \sigma, \beta)$ in $\mathfrak{Y} \times S^{\wedge} \times \mathscr{B}^{\wedge}$. From this observation both our above claims follow immediately.

By the following consideration we can get a parametrization of Priv $\left(U^{\wedge}, C^{*}\left(S \times U, C^{*}(N) \otimes \mathscr{B}, R\right), E\right)$ where $\mathfrak{Y}$ is replaced by $\mathfrak{g}_{\text {int }}^{*}$. Clearly, the map $\Phi^{\prime}$ factors through $\mathfrak{Q}^{\prime}=U \times S^{\wedge} \times \mathfrak{Y} \times \mathscr{B}^{\wedge} \rightarrow U \times S^{\wedge} \times \mathfrak{g}_{\text {int }}^{*} / N \times$ $\mathscr{B}^{\wedge}$ given by the map $\mathfrak{Y} \rightarrow \mathfrak{g}_{\text {int }}^{*} / N$ of Remark 1.21. Composing this factorized map with the most obvious projection from $U \times S^{\wedge} \times \mathfrak{g}_{\text {int }}^{*} \times \mathscr{B}^{\wedge}$ onto $U \times S^{\wedge} \times \mathfrak{g}_{\text {int }}^{*} / N \times \mathscr{B}^{\wedge}$ yields a surjection onto the primitive ideal space in question.

COROLlary 3.33. There is an open, continuous map $\Phi$ from $\mathfrak{Q}:=U \times S^{\wedge} \times$ 
$\mathfrak{g}_{\text {int }}^{*} \times \mathscr{B}^{\wedge}$ onto Priv $\left(U^{\wedge}, C^{*}\left(S \times U, C^{*}(N) \otimes \mathscr{B}, R\right), E\right):$ For $(u, \sigma, f, \beta) \in \mathfrak{Q}$ choose an $x \in N$ such that $\operatorname{Ad}^{*}(x) f$ is of the form $\operatorname{Ad}^{*}(x) f=f_{\omega, \lambda}$ with $(\omega, \lambda) \in U^{\wedge} \times \mathbf{R}^{\times}$in the sense of Proposition 1.18 or such that $\operatorname{Ad}^{*}(x) f=J(\omega, \gamma)$ with $(\omega, \gamma) \in U^{\wedge} \times(N / Z N)^{\wedge}$ in the sense of Lemma 1.15. iv depending on whether $\left.f\right|_{\mathfrak{z} \mathfrak{n}} \neq 0$ or $\left.f\right|_{\mathfrak{z} \mathfrak{n}}=0$. Then define $\Phi(u, \sigma, f, \beta)$ by $\Phi(u, \sigma, f, \beta)=\operatorname{ker}_{C^{*}} \chi_{u, \sigma, \omega, \mu, \beta}$ with $\mu=\lambda$ or $\mu=\gamma$. The equivalence relation $\approx$ defined by $\Phi$ can be described as follows. Introduce a group multiplication on $U^{\wedge} \times S \times G \times \mathrm{T} b y$

$$
\left(\omega_{1}, s_{1}, y_{1}, a_{1}\right)\left(\omega_{2}, s_{2}, y_{2}, a_{2}\right)=\left(\omega_{1} \omega_{2}, s_{1} s_{2}, y_{1} y_{2}, a_{1} a_{2} \omega_{2}\left(u_{1}^{-1} \alpha\left(s_{1}\right)\right)\right),
$$

where $u_{1}$ denotes the $U$-component of $y_{1} \in G=U \ltimes N$. This group acts on $\mathfrak{Q}$ via

$$
\left(\omega_{0}, s_{0}, y_{0}, a_{0}\right) \cdot(u, \sigma, f, \beta)=\left(u u_{0} \alpha\left(s_{0}\right)^{-1}, \sigma \widehat{\alpha}\left(\omega_{0}\right), \operatorname{Ad}^{*}\left(y_{0}\right) f-d \omega_{0}, s_{0} \cdot \beta\right),
$$

where $u_{0} \equiv y_{0} \bmod N$, and by the same formula on each quotient of $\mathfrak{Q}$ of the form $U \times\left(S^{\prime}\right)^{\wedge} \times \mathfrak{g}_{\mathrm{int}}^{*} \times \mathscr{B}^{\wedge}$ where $S^{\prime}$ is a closed subgroup of $S$. Then $(u, \sigma, f, \beta) \approx\left(u^{\prime}, \sigma^{\prime}, f^{\prime}, \beta^{\prime}\right)$ if and only if $\left.(S \beta)=S \beta^{\prime}\right)^{-}$, whence $S_{\beta}=S_{\beta^{\prime}}$, and the $U^{\wedge} \times S \times G \times$ T-quasi-orbits through $\left(u,\left.\sigma\right|_{S_{\beta}}, f, \beta\right)$ and $\left(u^{\prime},\left.\sigma^{\prime}\right|_{S_{\beta}}, f^{\prime}, \beta^{\prime}\right)$ coincide.

Proof. Since the map $\mathfrak{Y} \rightarrow \mathfrak{g}_{\mathrm{int}}^{*} / N$ of Remark 1.21 is continuous and open it follows immediately from Theorem 3.32 that $\Phi$ is continuous, open and onto. The proof for the description of $\approx$ is mainly left of the reader, because it is not difficult, but somewhat tedious and not too much enlightening. Perhaps it is more enlightening to observe that the surprisingly simple structure of $\approx$ reflects the fact that $C^{*}\left(U^{\wedge}, C^{*}\left(S \times U, C^{*}(N) \otimes\right.\right.$ $\mathscr{B}, R), E$ ) may also be viewed as a twisted covariance algebra on $U^{\wedge} \times S \times G \times$ T with values in $\mathscr{R}$.

But we do give some comments on the original description of $\approx$. For $(u, \sigma, f, \beta) \approx\left(u^{\prime}, \sigma^{\prime}, f^{\prime}, \beta^{\prime}\right)$ it is clearly necessary that $\left.f\right|_{\mathfrak{z} \mathfrak{n}}=\left.f^{\prime}\right|_{\mathfrak{z}_{\mathfrak{n}}}$. In the case $\left.f\right|_{\mathfrak{z} \mathfrak{n}}=\left.f^{\prime}\right|_{\mathfrak{z} \mathfrak{n}} \neq 0$ choose $x, x^{\prime} \in N$ such that $\operatorname{Ad}^{*}(x) f=f_{\omega, \lambda}$ and $\operatorname{Ad}^{*}\left(x^{\prime}\right) f^{\prime}=$ $f_{\omega^{\prime}, \lambda}$ in the sense of Proposition 1.18. The elements $x$ and $x^{\prime}$ are uniquely determined modulo $Z N$, in particular $\omega$ and $\omega^{\prime}$ are well-defined. Here one has $(u, \sigma, f, \beta) \approx\left(u^{\prime}, \sigma^{\prime}, f^{\prime}, \beta^{\prime}\right)$ if and only if $(S \beta)^{-}=\left(S \beta^{\prime}\right)^{-}$and $\left.\widehat{\alpha}\left(\omega^{\prime}\right) \sigma^{\prime}\right|_{S_{\beta}}=$ $\left.\widehat{\alpha}(\omega) \sigma\right|_{S_{\beta}}$. In the case $\left.f\right|_{\mathfrak{z} \mathfrak{n}}=0=\left.f^{\prime}\right|_{\mathfrak{z} \mathfrak{n}}$ choose $x, x^{\prime} \in N$ such that

$$
\operatorname{Ad}^{*}(x) f=d \omega+d \gamma \quad \text { and } \quad \operatorname{Ad}^{*}\left(x^{\prime}\right) f^{\prime}=d \omega^{\prime}+d \gamma^{\prime}
$$

for some $\omega, \omega^{\prime} \in U^{\wedge}, \gamma, \gamma^{\prime} \in(N / Z N)^{\wedge}$. The character $\gamma$ is uniquely determined, namely $d \gamma=\left.f\right|_{\mathfrak{n}}$, the $\omega$ is not, but $\left.\omega\right|_{U_{\gamma}}$ still is because $U_{\gamma}$ is connected and $d \omega=f$ on Lie $\left(U_{\gamma}\right)$, compare Lemma 1.15. Here one has 
$(u, \sigma, f, \beta) \approx\left(u^{\prime}, \sigma^{\prime}, f^{\prime}, \beta^{\prime}\right)$ if and only if the $S \times U$-quasi-orbits through $(u, \gamma, \beta)$ and $\left(u^{\prime}, \gamma^{\prime}, \beta^{\prime}\right)$ coincide for the action $\left(s_{0}, u_{0}\right) \cdot(u, \gamma, \beta)=$ $\left(u_{0} \alpha\left(s_{0}\right)^{-1} u, u_{0} \cdot \gamma, s_{0} \cdot \beta\right)$, whence $S_{\beta}=S_{\beta^{\prime}}, U \gamma=U \gamma^{\prime}$ and $U_{\gamma}=U_{\gamma^{\prime}}$, and if $\sigma^{\prime}(s) \omega^{\prime}(\alpha(s))=\sigma(s) \omega(\alpha(s))$ for all $s \in S_{\beta}$ with $\alpha(s) \in U_{\gamma}$. Observe that the description (3.20) of $\sim$ is here more convenient than the modified form (3.21). With this information at hand it is not hard to write down a formal proof using in addition Lemma 1.15 and that elements of the form $f_{\omega, \lambda}$ are fixed by $U$.

The equivalence relation $\approx$ is even more pleasant in the "group case", by which we mean that $\mathscr{B}$ is the $C^{*}$-algebra of a second countable locally compact abelian group $B$ and $S$ acts on $L^{1}(B) \subset C^{*}(B)$ by $\left(T_{s} h\right)(b)=$ $h(b) \widehat{\eta}(s)(b)^{-1}$ where $\widehat{\eta}$ is the dual of a continuous homomorphism $\eta: B \rightarrow S^{\wedge}$, compare (3.2). Then there is an "orbit picture", the equivalence classes are quasi-orbits of the auxiliary group $H$ which as a space is $U^{\wedge} \times S \times G \times B \times \mathrm{T}$, and the multiplication is defined by

$$
\begin{aligned}
& \left(\omega_{1}, s_{1}, y_{1}, b_{1}, a_{1}\right)\left(\omega_{2}, s_{2}, y_{2}, b_{2}, a_{2}\right)= \\
& \left(\omega_{1} \omega_{2}, s_{1} s_{2}, y_{1} y_{2}, b_{1} b_{2}, a_{1} a_{2} \omega_{2}\left(u_{1}^{-1} \alpha\left(s_{1}\right)\right) \widehat{\eta}\left(s_{2}\right)\left(b_{1}\right)\right),
\end{aligned}
$$

where $u_{1} \equiv y_{1} \bmod N$, i.e., $H$ is a central extension of the direct product $U^{\wedge} \times S \times G \times B$. Here our object of study $C^{*}\left(U^{\wedge}, C^{*}\left(S \times U, C^{*}(N \times B)\right.\right.$, $R), E)$ is just $C^{*}(H)_{\mathrm{Id}}$ which is the $C^{*}$-completion of the convolution algebra consisting of all measurable functions $\varphi: H \rightarrow \mathrm{C}$ with $\varphi(x a)=\varphi(x) \bar{a}$ for all $x \in H, a \in \mathrm{T}$ and $\int_{H / \mathrm{T}}|\varphi(x)| d \dot{x}<\infty$. The group $H$ acts by conjugation on itself via

$$
\begin{aligned}
& \left(\omega_{0}, s_{0}, y_{0}, b_{0}, a_{0}\right)^{-1}(\omega, s, y, b, a)\left(\omega_{0}, s_{0}, y_{0}, b_{0}, a_{0}\right)= \\
& \left(\omega, s, y_{0}^{-1} y y_{0}, b, a \omega\left(u_{0} \alpha\left(s_{0}\right)^{-1}\right) \widehat{\eta}(s)\left(b_{0}\right)^{-1} \omega_{0}\left(u^{-1} \alpha(s)\right) \widehat{\eta}\left(s_{0}\right)(b)\right),
\end{aligned}
$$

where $u \equiv y, u_{0} \equiv y_{0} \bmod N$, and accordingly on its "linear dual" $\mathfrak{h}^{*}:=$ $U \times S^{\wedge} \times \mathfrak{g}_{\text {int }}^{*} \times B^{\wedge}$ via

$$
\begin{aligned}
& \left(\omega_{0}, s_{0}, y_{0}, b_{0}, a_{0}\right) \cdot(u, \sigma, f, \beta)= \\
& \left(u u_{0} \alpha\left(s_{0}\right)^{-1}, \sigma \eta\left(b_{0}\right)^{-1} \widehat{\alpha}\left(\omega_{0}\right), \operatorname{Ad}^{*}\left(y_{0}\right) f-d \omega_{0}, \beta \widehat{\eta}\left(s_{0}\right)\right) .
\end{aligned}
$$

The space $\mathfrak{Q}$ for the present case is nothing but $\mathfrak{h}^{*}$.

COROllary 3.36. If $\mathscr{B}=C^{*}(B)$ for a second countable locally compact abelian group $B$, and if $S$ acts on $\mathscr{B}$ according to (3.2) then the equivalence classes of the relation $\approx$ of Corollary 3.33 are precisely the $H$-quasi-orbits in the above $H$-space $\mathfrak{h}^{*}$, hence Priv $\left(U^{\wedge}, C^{*}\left(S \times U, C^{*}(N \times B), R\right), E\right)$ is homeomorphic to the $H$-quasi-orbit space $\mathfrak{h}^{*} / H$. 
Proof. This corollary follows immediately from (3.33) by observing that the $S$-action on $\mathscr{B}^{\wedge}=B^{\wedge}$ is given by $s \cdot \beta=\beta \widehat{\eta}(s)$, whence all the stabilizers $S_{\beta}$ coincide with ker $\widehat{\eta}$, and that the closure of $\eta(B)$ in $S^{\wedge}$ is nothing but $(\operatorname{ker} \widehat{\eta})^{\perp}=S_{\beta}^{\perp}=\left(S / S_{\beta}\right)^{\wedge} \subset S^{\wedge}$.

We conclude this section by returning to the original algebra $C^{*}\left(S, C^{*}(N) \otimes \mathscr{B}, T\right)$. Having in mind the topological space $\mathfrak{Y}$ we introduce a (non Hausdorff) topology on the disjoint union of $S^{\wedge} \times \mathrm{R}^{\times}$and $S^{\wedge} \times(N / Z N)^{\wedge}$, which should not be confused with the topology introduced in Proposition 1.18 on a similar set. Actually, comparing these two topologies may be regarded as a central issue of this article. The subset $S^{\wedge} \times \mathrm{R}^{\times}$is open and carries the product topology. A base for the neighborhood system of $(\sigma, \gamma) \in S^{\wedge} \times(N / Z N)^{\wedge}$ is given by the collection of sets $W_{\varepsilon}(\sigma, \gamma, V)$, $\varepsilon>0, V$ an open neighborhood of $\sigma$ in $S^{\wedge}$, where $W_{\varepsilon}(\sigma, \gamma, V)$ is the union of the set of all pairs $(\tau, \mu) \in S^{\wedge} \times(N / Z N)^{\wedge}$ with $\tau \in V$ and $\left|\gamma_{j}-\mu_{j}\right|<\varepsilon$ for $1 \leq j \leq n$ where $d \gamma(z)=\sum_{j=1}^{n} \operatorname{Im}\left(\gamma_{j} \bar{z}_{j}\right)$ and $d \mu(z)=\sum_{j=1}^{n} \operatorname{Im}\left(\mu_{j} \bar{z}_{j}\right)$ for $z \in \mathrm{C}^{n}$, and of the set of all pairs $(\tau, \lambda) \in S^{\wedge} \times \mathbf{R}^{\times}$such that $|\lambda|<\varepsilon$ and there exists $m=\left(m_{1}, \ldots, m_{n}\right) \in Z^{n} \quad$ with $\quad \tau \prod_{j=1}^{n} \widehat{\alpha}\left(\zeta_{j}^{-1}\right)^{m_{j}} \in V, \quad m_{j} \lambda \geq 0 \quad$ and $\left|\left(2 m_{j} \lambda\right)^{\frac{1}{2}}-\right| \gamma_{j}||<\varepsilon$ for $1 \leq j \leq n$. Recall that $\zeta_{j}$ was introduced in (1.3).

It is not hard to verify that the topology is well-defined. Concerning convergence we observe the following.

(3.37) A sequence $\left(\sigma_{k}, \lambda_{k}\right)$ in $S^{\wedge} \times \mathrm{R}^{\times}$converges to $(\sigma, \gamma) \in S^{\wedge} \times(N / Z N)^{\wedge}$ if and only if $\left(\lambda_{k}\right)$ converges to zero and if there exists a sequence $m_{k} \in \mathbf{Z}^{n}$ such that $\lambda_{k} m_{k}^{(j)} \geq 0$ for $1 \leq j \leq n$ and all sufficiently large $k$, that the sequence $\sigma_{k} \prod_{j=1}^{n} \widehat{\alpha}\left(\zeta_{j}^{-1}\right)^{m_{k}^{(j)}}$ converges to $\sigma$ in $S^{\wedge}$, and that $2 m_{k}^{(j)} \lambda_{k}$ converges to $\left|\gamma_{j}\right|^{2}$ for $1 \leq j \leq n$.

(3.38) The set $\mathfrak{P}:=S^{\wedge} \times \mathrm{R}^{\times} \times \mathscr{B}^{\wedge} \cup S^{\wedge} \times(N / Z N)^{\wedge} \times \mathscr{B}^{\wedge}=\left(S^{\wedge} \times\right.$ $\left.\mathrm{R}^{\times} \cup S^{\wedge} \times(N / Z N)^{\wedge}\right) \times \mathscr{B}^{\wedge}$ is equipped with with the product topology. Recall that $\mathfrak{P}$ parametrizes Priv $\left(S, C^{*}(N) \otimes \mathscr{B}, T\right)$.

THEOREM 3.39. Let $(S, \mathscr{B})$ be a covariance pair with action $\mathscr{B} \times S \ni$ $(b, s) \mapsto b^{s} \in \mathscr{B}$, where $S$ is a second countable locally compact abelian group, and $\mathscr{B}$ is a separable commutative $C^{*}$-algebra. For a continuous homomorphism $\alpha: S \rightarrow U$ define an action $T$ of $S$ on $C^{*}(N) \otimes \mathscr{B}$ by

$$
\left(T_{s} h\right)(x)=h\left(\alpha(s)^{-1} x\right)^{s^{-1}}
$$

for $h \in L^{1}(N, \mathscr{B}) \subset C^{*}(N) \otimes \mathscr{B}$. Then the map $\Psi$ from the above $\mathfrak{P}$ into $\operatorname{Priv}\left(S, C^{*}(N) \otimes \mathscr{B}, T\right)$ defined by $\Psi(\sigma, \mu, \beta)=\operatorname{ker}_{C^{*}} \pi_{\sigma, \mu, \beta} \quad$ for $\quad \sigma \in S^{\wedge}$, $\beta \in \mathscr{B}^{\wedge}, \mu \in \mathbf{R}^{\times} \cup(N / Z)^{\wedge}$, the representations $\pi_{\sigma, \mu, \beta}$ being given in $(3.5 / 8)$, is continuous, open and onto. The equivalence relation $\sim$ on $\mathfrak{P}$ defined by $\Psi$ is given in (3.6/9) i.e., $\Psi(\sigma, \mu, \beta)=\Psi\left(\sigma^{\prime}, \mu^{\prime}, \beta^{\prime}\right)$ if and only if either $\mu$ and $\mu^{\prime}$ are 
in $\mathrm{R}^{\times}$, in which case $\mu=\mu^{\prime},(S \beta)^{-}=\left(S \beta^{\prime}\right)^{-}$and $\left.\sigma\right|_{S_{\beta}}=\left.\sigma^{\prime}\right|_{S_{\beta^{\prime}}}$ or $\mu$ and $\mu^{\prime}$ are in $(N / Z)^{\wedge}$, in which case $(S \cdot(\mu, \beta))^{-}=\left(S \cdot\left(\mu^{\prime}, \beta^{\prime}\right)\right)^{-}$and $\left.\sigma\right|_{S_{\mu} \cap S_{\beta}}=\left.\sigma^{\prime}\right|_{S_{\mu^{\prime}} \cap S_{\beta^{\prime}}}$.

Proof. In view of the established relation between Priv $\left(S, C^{*}(N) \otimes\right.$ $\mathscr{B}, T)$ and the associated space Priv $\left(U^{\wedge}, C^{*}\left(S \times U, C^{*}(N) \otimes \mathscr{B}, R\right), E\right)$, compare $(3.25 / 28 / 30 / 31)$, and in view of Theorem 3.32 we only have to show that the composition $I^{\prime}$ of $I: \mathfrak{P} \rightarrow \mathfrak{Q}^{\prime}=U \times S^{\wedge} \times \mathfrak{Y} \times \mathscr{B}^{\wedge}$ and the quotient map $\mathfrak{Q}^{\prime} \rightarrow \mathfrak{Q}^{\prime} / \sim$ is continuous, open and onto. As we remarked earlier this map is onto and behaves well on the two pieces of $\mathfrak{P}$. Therefore, to prove the continuity of $I^{\prime}$ it suffices to show that for a sequence $\left(\sigma_{k}, \lambda_{k}, \beta_{k}\right)$ in $S^{\wedge} \times \mathbf{R}^{\times} \times \mathscr{B}^{\wedge}$ converging to $(\sigma, \gamma, \beta) \in S^{\wedge} \times(N / Z N)^{\wedge} \times \mathscr{B}^{\wedge}$ there exist $q_{k}^{\prime} \in \mathfrak{Q}^{\prime}$ with $q_{k}^{\prime} \sim I\left(\sigma_{k}, \lambda_{k}, \beta_{k}\right)=\left(1, \sigma_{k}, 1, \lambda_{k}, \beta_{k}\right)$ such that $\left(q_{k}^{\prime}\right)$ converges to $I(\sigma, \gamma, \beta)=(1, \sigma, 1, \gamma, \beta)$. As $\left(\sigma_{k}, \lambda_{k}, \beta_{k}\right)$ converges to $(\sigma, \gamma, \beta)$ we know that $\lim _{k \rightarrow \infty} \beta_{k}=\beta, \lim _{k \rightarrow \infty} \lambda_{k}=0$, and that there exists a sequence $m_{k} \in Z^{n}$ such that $\lim _{k \rightarrow \infty} \sigma_{k}^{\prime}=\sigma$, where $\sigma_{k}^{\prime}:=\sigma_{k} \prod_{j=1}^{n} \widehat{\alpha}\left(\zeta_{j}^{-1}\right)^{m_{k}^{(j)}}, \lambda_{k} m_{k}^{(j)} \geq 0$ for large $k$ and $\lim _{k \rightarrow \infty} 2 m_{k}^{(j)} \lambda_{k}=\left|\gamma_{j}\right|^{2}$ for $1 \leq j \leq n$. Clearly, we choose $q_{k}^{\prime}=\left(1, \sigma_{k}^{\prime}, \omega_{k}^{\prime}, \lambda_{k}, \beta_{k}\right)$, where $\omega_{k}^{\prime}=\prod_{j=1}^{n} \zeta_{j}^{m_{k}^{(j)}}$. It is easy to check that $q_{k}^{\prime}$ is equivalent to $I\left(\sigma_{k}, \lambda_{k}, \beta_{k}\right)$ in the sense of (3.23), and from the definition of the topology on $\mathfrak{Y}$ it readily follows that $\left(q_{k}^{\prime}\right)$ converges to $I(\sigma, \gamma, \beta)$.

Secondly to see the openness of $I^{\prime}$ in view of the above remarks and of Lemma 1.9 it suffices to show that if for a given sequence $\left(\sigma_{k}, \lambda_{k}, \beta_{k}\right)$ in $S^{\wedge} \times \mathrm{R}^{\times} \times \mathscr{B}^{\wedge}$ there exists a sequence $\left(q_{k}^{\prime}\right)$ in $\mathfrak{Q}^{\prime}$ such that $q_{k}^{\prime} \sim I\left(\sigma_{k}, \lambda_{k}, \beta_{k}\right)$ and that $\left(q_{k}^{\prime}\right)$ converges to $I(\sigma, \gamma, \beta)$ for some $(\sigma, \gamma, \beta) \in S^{\wedge} \times$ $(N / Z N)^{\wedge} \times \mathscr{B}^{\wedge}$ that then there is a sequence $\left(\sigma_{k}^{\prime \prime}, \lambda_{k}, \beta_{k}^{\prime \prime}\right)$ in $\mathfrak{P}$, $\left(\sigma_{k}^{\prime \prime}, \lambda_{k}, \beta_{k}^{\prime \prime}\right) \sim\left(\sigma_{k}, \lambda_{k}, \beta_{k}\right)$, such that $\left(\sigma_{k}^{\prime \prime}, \lambda_{k}, \beta_{k}^{\prime \prime}\right)$ converges to $(\sigma, \gamma, \beta)$. As $q_{k}^{\prime}=\left(\mu_{k}^{\prime}, \sigma_{k}^{\prime}, \omega_{k}^{\prime}, \lambda_{k}, \beta_{k}^{\prime}\right)$ is equivalent to $I\left(\sigma_{k}, \lambda_{k}, \beta_{k}\right)$ we know that $\left(S \beta_{k}^{\prime}\right)^{-}=\left(S \beta_{k}\right)^{-}$and $\left.\widehat{\alpha}\left(\omega_{k}^{\prime}\right) \sigma_{k}^{\prime}\right|_{S_{\beta_{k}}}=\left.\sigma_{k}\right|_{S_{\beta_{k}}}$. Since $\left(q_{k}^{\prime}\right)$ converges to $I(\sigma, \gamma, \beta)$ we conclude that $\lim _{k \rightarrow \infty} \beta_{k}^{\prime}=\beta$, $\lim _{k \rightarrow \infty} \sigma_{k}^{\prime}=\sigma$, and that $\left(\omega_{k}^{\prime}, \lambda_{k}\right)$ converges in $\mathfrak{Y}$ to $(1, \gamma)$. The desired $\beta_{k}^{\prime \prime}, \sigma_{k}^{\prime \prime}$ are obtained by choosing $\beta_{k}^{\prime \prime}=\beta_{k}^{\prime}$ and $\sigma_{k}^{\prime \prime}=\widehat{\alpha}\left(\omega_{k}^{\prime}\right) \sigma_{k}^{\prime}$. From the definition of the topologies on $\mathfrak{Y}$ and on $\mathfrak{P}$ it readily follows that $\left(\sigma_{k}^{\prime \prime}, \lambda_{k}, \beta_{k}^{\prime \prime}\right)$ converges to $(\sigma, \gamma, \beta)$.

\section{$\S$ 4. Special Cases}

In this section we study some special cases of diamond groups $S \ltimes N$ in the sense of Definition 1.4. The above considered algebra $\mathscr{B}$ reduces to $\mathrm{C}$, and the parameter space $\mathfrak{P}$ reduces to $S^{\wedge} \times \mathrm{R}^{\times} \cup S^{\wedge} \times(N / Z N)^{\wedge}$. The map $\Psi: \mathfrak{P} \rightarrow \operatorname{Priv}(S \ltimes N)$ of Theorem 3.39 is given by

(4.1) $\Psi(\sigma, \mu)=\operatorname{ker}_{C^{*}(S \ltimes N)} \pi_{\sigma, \mu}$ for $\sigma \in S^{\wedge}, \mu \in \mathrm{R}^{\times}$or $\mu \in(N / Z N)^{\wedge}$ where $\pi_{\sigma, \gamma}, \gamma \in(N / Z N)^{\wedge}$, is the unitary representation of $S \ltimes N$ in $L^{2}\left(S / S_{\gamma}\right)$ defined by 


$$
\left(\pi_{\sigma, \gamma}(s, z, t) \xi\right)(r)=\sigma(s) \gamma\left(\alpha\left(r^{-1} s\right)(z)\right) \xi\left(s^{-1} r\right),
$$

and $\pi_{\sigma, \lambda}, \lambda \in \mathrm{R}, \lambda \neq 0$ is the unitary representation of $S \ltimes N$ in $\mathfrak{F}_{\lambda}$ defined by

$$
\begin{aligned}
& \left(\pi_{\sigma, \lambda}(s, z, t) \xi\right)(w)= \\
& \sigma(s) e^{i \lambda t-\frac{1}{2} \lambda \alpha\left(s^{-1}\right)(w) \bar{z}-\frac{1}{4} \lambda|z|^{2}} \xi\left(z+\alpha\left(s^{-1}\right)(w)\right) \text { if } \quad \lambda>0
\end{aligned}
$$

or

$$
\begin{aligned}
& \left(\pi_{\sigma, \lambda}(s, z, t) \xi\right)(w)= \\
& \sigma(s) e^{i \lambda t+\frac{1}{2} \lambda \alpha(s)(w) z+\frac{1}{4} \lambda|z|^{2}} \xi(\alpha(s) w+\bar{z}) \quad \text { if } \quad \lambda<0,
\end{aligned}
$$

i.e., $\pi_{\sigma, \lambda}=\sigma \otimes\left(\pi_{1, \lambda}^{\prime} \circ \alpha^{\prime}\right)$ if $\alpha^{\prime}: S \ltimes N \rightarrow U \ltimes N$ is the obvious homomorphism, and $\pi_{1, \lambda}^{\prime}$ denotes the representation of $U \ltimes N$ defined in (2.5).

Also the equivalence relation $\sim$ on $\mathfrak{P}$ simplifies. The only (possibly) not identical, but equivalent pairs are such of the form $(\sigma, \gamma),\left(\sigma^{\prime}, \gamma^{\prime}\right) \in$ $S^{\wedge} \times(N / Z N)^{\wedge}$ with $(S \gamma)^{-}=\left(S \gamma^{\prime}\right)^{-}$and $\left.\sigma\right|_{S_{\gamma}}=\left.\sigma^{\prime}\right|_{S_{\gamma}}$. The condition $(S \gamma)^{-}=\left(S \gamma^{\prime}\right)^{-}$can be rephrased by saying that $\gamma$ and $\gamma^{\prime}$ are on the same $\alpha(S)^{-}$-orbit where $\alpha(S)^{-}$is the closure of $\alpha(S)$ in $U$.

Even though we are primarily interested in connected Lie groups we start with some remarks on the case of finite groups $S$. We wish to derive a necessary and sufficient condition for a sequence $\left(\pi_{k}\right)$ in $(S \ltimes N)^{\wedge}$ to converge to a given $\pi \in(S \ltimes N)^{\wedge}$. In the present case it is not a severe restriction to assume that $\alpha: S \rightarrow U$ is faithful: For general $\alpha$ the kernel of $\alpha$ is compact (finite) and central, hence $\pi_{k}$ restricts to a sequence of characters of $\operatorname{ker} \alpha$, which eventually has to be constant, equal to $\left.\pi\right|_{\operatorname{ker} \alpha}$.

Then $\widehat{\alpha}: U^{\wedge} \rightarrow S^{\wedge}$ is surjective, for a sufficiently large fixed $M \in \mathrm{N}$ the kernel of $\widehat{\alpha}$ contains $\left\{\zeta_{1}^{m_{1}} \cdot \ldots \cdot \zeta_{n}^{m_{n}} \mid M\right.$ divides each $\left.m_{j}\right\}$, and each $\sigma \in S^{\wedge}$ can be written as $\sigma=\widehat{\alpha}\left(\zeta_{1}^{m_{1}} \cdot \ldots \cdot \zeta_{n}^{m_{n}}\right)$ with $\left|m_{j}\right| \leq M$ for $1 \leq j \leq n$. Let's consider any sequence $\left(\sigma_{k}, \lambda_{k}\right)$ in $S^{\wedge} \times \mathrm{R}^{\times}$. For such a sequence to converge to a point in $S^{\wedge} \times(N / Z N)^{\wedge}$ it is clearly necessary that $\left(\lambda_{k}\right)$ tends to zero. We claim that then $\left(\sigma_{k}, \lambda_{k}\right)$ converges to any point of $S^{\wedge} \times(N / Z N)^{\wedge}$, i.e., $\left(\pi_{\sigma_{k}, \lambda_{k}}\right)$ converges to any $\pi_{\sigma, \gamma}, \gamma \in(N / Z N)^{\wedge}$, independent of the behaviour of $\sigma_{k}$.

For a given $(\sigma, \gamma), d \gamma(z)=\sum_{j=1}^{n} \operatorname{Im} \gamma_{j} \overline{z_{j}}$ for $z \in \mathbf{C}^{n}$, choose $m_{k}^{\prime} \in \mathbf{Z}^{n}$, $\left|m_{k}^{\prime(j)}\right| \leq M$ for $1 \leq j \leq n$, such that

$$
\sigma_{k} \sigma^{-1}=\prod_{j=1}^{n} \widehat{\alpha}\left(\zeta_{j}\right)^{m^{\prime}{ }_{k}^{(j)}} .
$$

Then modify $m_{k}^{\prime}$ to $m_{k}=m_{k}^{\prime}+m_{k}^{\prime \prime}$ where $m_{k}^{\prime \prime(j)}=\operatorname{sign}\left(\lambda_{k}\right) M$ if $\gamma_{j}=0$, and for $\gamma_{j} \neq 0$ the $m_{k}^{\prime \prime(j)}$ are chosen to be any integral multiples of $M$ such that $2 \lambda_{k} m_{k}^{\prime \prime(j)}$ converges to $\left|\gamma_{j}\right|^{2}$. It is clear that this choice delivers a sequence $\left(m_{k}\right)$ needed for the convergence criterion (3.37). 
For the study of connected Lie diamond groups we first introduce some further notation. Define $\Lambda: \mathfrak{P} \rightarrow(\mathfrak{s} \ltimes \mathfrak{n})_{\text {int }}^{*}$, for the latter set compare Definition 1.10 , by

$$
\Lambda(\sigma, \lambda)(A, z, t)=d \sigma(A)+\lambda t \text { if } \sigma \in S^{\wedge} \text { and } \lambda \in \mathbf{R}, \lambda \neq 0,
$$

and by $\quad \Lambda(\sigma, \gamma)(A, z, t)=d \sigma(A)+d \gamma(z)$ if $\sigma \in S^{\wedge}$ and $\gamma \in(N / Z N)^{\wedge}$,

i.e. on the subset $S^{\wedge} \times(N / Z N)^{\wedge}$ the map $\Lambda$ is nothing but the formerly considered $J$, compare Lemma 1.15 . Denote by $\Lambda^{\prime}$ the composition on $\Lambda$ with the canonical projection from $(\mathfrak{s} \ltimes \mathfrak{n})_{\text {int }}^{*}$ onto the $(S \ltimes N)$-quasi-orbit space $(\mathfrak{s} \ltimes \mathfrak{n})_{\text {int }}^{*} / S \ltimes N$. The map $\Lambda^{\prime}$ factors through the equivalence relation $\sim$ on $\mathfrak{P}$ and gives rise to a map

$$
\Omega: \operatorname{Priv}(S \ltimes N) \rightarrow(\mathfrak{s} \ltimes \mathfrak{n})_{\text {int }}^{*} / S \ltimes N \text { with } \Omega \circ \Psi=\Lambda^{\prime} .
$$

The map $\Omega$, or more precisely its factorized version, factorized through the appropriate equivalence relation on $\operatorname{Priv}(S \ltimes N)$, is nothing but the inverse of the Pukanszky-Kirillov map mentioned in the introduction.

Theorem 4.6 (compare [14]). For connected Lie diamond groups $S \ltimes N$ the above map $\Omega$ is continuous.

Proof. Because of Theorem 3.39 we only have to show that $\Lambda^{\prime}$ is continuous. In view of $(1.15 / 18)$ and by the definition of the topology on $\mathfrak{P}$ it suffices to prove that for a sequence $\left(\sigma_{k}, \lambda_{k}\right)$ in $S^{\wedge} \times \mathbf{R}^{\times}$, a sequence $\left(m_{k}\right)$ in $Z^{n}$, and a point $(\sigma, \gamma) \in S^{\wedge} \times(N / Z N)^{\wedge}$ such that $\left(\lambda_{k}\right)$ converges to zero, $m_{k}^{(j)} \lambda_{k} \geq 0$ for $1 \leq j \leq n$ and all sufficiently large $k, \lim _{k \rightarrow \infty} \sigma_{k}$ $\prod_{j=1}^{n} \widehat{\alpha}\left(\zeta_{j}\right)^{-m_{k}^{(j)}}=\sigma$, and $\lim _{k \rightarrow \infty} 2 m_{k}^{(j)} \lambda_{k}=\left|\gamma_{j}\right|^{2}$ for $1 \leq j \leq n$, where as usual $d \gamma(z)=\sum_{j=1}^{n} \operatorname{Im}\left(\gamma_{j} \bar{z}_{j}\right)$, there exists a sequence $\left(w_{k}\right)$ in $\mathrm{C}^{n}$ with limit $\left(\gamma_{1}, \ldots, \gamma_{n}\right)$ such that $d \sigma=\lim _{k \rightarrow \infty} d \sigma_{k}-\frac{1}{2 \lambda_{k}} \sum_{j=1}^{n}\left|w_{k}^{(j)}\right|^{2} d \alpha_{j}$. But as in the proof of (1.18.iv) such a sequence is easily constructed by putting $w_{k}^{(j)}=\left(2 \lambda_{k} m_{k}^{(j)}\right)^{\frac{1}{2}}$ if $\gamma_{j}=0$, and $w_{k}^{(j)}=\left(2 \lambda_{k} m_{k}^{(j)}\right)^{\frac{1}{2}} \frac{\gamma_{j}}{\left|\gamma_{j}\right|}$ if $\gamma_{j} \neq 0$.

However, in general for simply connected Lie diamond groups $S \ltimes N$ the above map $\Omega$ is not open, even worse, neither is the equivalence relation on Priv $(S \ltimes N)$ defined by $\Omega$ open, nor does the quasi-orbit-space $(\mathfrak{s} \ltimes \mathfrak{n})^{*} / S \ltimes N$ carry the quotient topology w.r.t. $\Omega$.

This happens already in the example of the least possible dimension, i.e., $N=N_{1}=\mathrm{C} \times \mathrm{R}$, and $\alpha: S=\mathrm{R} \rightarrow U=\mathrm{T}$ is the universal covering. For the first claim, as $\Psi: \mathfrak{P} \rightarrow \operatorname{Priv}(S \ltimes N)$ is open and continuous it suffices to show that the equivalence relation on $\mathfrak{P}$ defined by $\Lambda^{\prime}$ is not open. Consider for instance the subset 


$$
X=\left\{\left(\widehat{\alpha}\left(\zeta^{k}\right), \frac{1}{2 k}\right) \mid k \in \mathrm{N}\right\}
$$

of $\mathfrak{P}$. The closure $X^{-}$in $\mathfrak{P}$ is the union of $X$ and $\left\{\left(\widehat{\alpha}\left(\zeta^{m}\right), \gamma\right) \mid m \in \mathbf{Z}\right.$, $\left.\gamma \in(N / Z N)^{\wedge} \cong \mathrm{C},|\gamma|=1\right\}$. The set $X$ is saturated w.r.t. the equivalence defined by $\Lambda^{\prime}$ (which is the identity on $S^{\wedge} \times \mathrm{R}^{\times}$), but its closure is not: All points in $\left\{(\sigma, \gamma)\left|\sigma \in S^{\wedge}, \gamma \in(N / Z N)^{\wedge} \cong \mathrm{C},\right| \gamma \mid=1\right\}$ are equivalent to points in $X^{-}$, compare Remark 1.16. This means that the dual version of the definition of open equivalence relations does not hold.

For the second claim, again as $\Psi$ is open and continuous, it suffices to show that $(\mathfrak{s} \ltimes \mathfrak{n})^{*} / S \ltimes N$ does not carry the quotient topology w.r.t. $\Lambda^{\prime}$. Take any $\sigma \in S^{\wedge}$ and put $\tau=\sigma \widehat{\alpha}(\zeta)^{\frac{1}{2}}$. Then the union $Y$ of $\left\{\left(\tau, \frac{1}{k}\right) \mid k \in \mathrm{N}\right\} \subset$ $S^{\wedge} \times \mathbf{R}^{\times}$and $\left\{\left(\tau \widehat{\alpha}\left(\zeta^{-1}\right)^{m}, 0\right) \mid m \in \mathrm{N}_{0}\right\} \subset S^{\wedge} \times(N / Z)^{\wedge}$, where 0 stands for the constant character, is closed in $\mathfrak{P}$ and saturated w.r.t. the equivalence relation defined by $\Lambda^{\prime}$. But in the closure of the image $\Lambda^{\prime}(Y)$ one finds for instance (this is very easy) the orbit $\{d \sigma\}$, where of course $d \sigma$ denotes the functional $d \sigma(A, z, t)=d \sigma(A)$, compare also Proposition 1.18.

Translated into terms of representation theory, there are sequences $\left(f_{k}\right)$ of functionals on $\mathfrak{s} \ltimes \mathfrak{n}$, for instance $f_{k}(A, z, t)=d \sigma(A)+\frac{1}{2} d \alpha(A)+\lambda_{k} t$ for any sequence $\left(\lambda_{k}\right)$ of positive real numbers converging to zero, and functionals $f_{k}^{\prime}$ in the $N$-orbit through $f_{k}$ such that $\left(f_{k}^{\prime}\right)$ converges to $d \sigma$ while the representations $\pi_{k}$ attached to the orbit through $f_{k}$ (observe that they are uniquely determined by the orbit as the stabilizers of the $f_{k}$ are connected, no additional parameters like in [19] are required here) don't converge to $\sigma$ (considered as a one-dimensional representation of $S \ltimes N$ ).

REMARK 4.7. Concerning the additional parameters, which are unitary characters $\vartheta_{k}^{\prime}$ on the stabilizer groups $(S \ltimes N)_{f_{k}^{\prime}}$, the following behaviour is conspicuous. While the $(S \ltimes N)_{f_{k}^{\prime}}$ are connected two-dimensional subgroups being conjugate to $(S \ltimes N)_{f_{k}}=S \times Z N$ the sequence $(S \ltimes N)_{f_{k}^{\prime}}$, or more precisely any convergent subsequence, in Fell's space $\Sigma(S \ltimes N)$ of closed subgroups of $S \ltimes N$ converges to $\operatorname{ker} \alpha \times M$ where $M$ is a connected two-dimensional subgroup of $N$; a closely related example was considered in [11, Example 23]. Even though the characters $\vartheta_{k}^{\prime}$ are completely determined by the convergent sequence $f_{k}^{\prime}$ very natural conditions for convergence (in the spirit of (1.14) can be imposed on the Pukanszky parameters, which ensure that the present sequence $\vartheta_{k}^{\prime}$ diverges - as one hopes for. In the Example 4.12 below the situation is different.

Motivated by the satisfactory picture for the unitary dual of the full diamond group we now study diamond groups $S \ltimes N$ where $S$ is a torus. As in the finite case it is not a severe restriction to assume that $\alpha$ is injective. Then 
the group $\mathfrak{s}_{\text {int }}^{*}$ is generated by $d \alpha_{1}, \ldots, d \alpha_{n}$. Moreover, the functionals $d \alpha_{1}, \ldots, d \alpha_{n}$ generate a cone $C$ in $\mathfrak{s}^{*}$,

$$
C=\left\{\sum_{j=1}^{n} r_{j} d \alpha_{j} \mid r_{j} \in \mathrm{R}, r_{j} \geq 0\right\},
$$

and there is also a "discrete" version of $C$, namely

$$
C_{d}:=\left\{\sum_{j=1}^{n} m_{j} d \alpha_{j} \mid m_{j} \in \mathrm{N}_{0}\right\} .
$$

Evidently, one has $C_{d} \subset C \cap \mathfrak{s}_{\text {int }}^{*}$.

THEOREM 4.10. Let $S$ be a torus, assume that $\alpha: S \rightarrow U$ is injective, and that $C, C_{d}$ and $\mathfrak{s}_{\text {int }}^{*}$ are as above. Then the map $\Omega: \operatorname{Priv}(S \ltimes N) \rightarrow$ $(\mathfrak{s} \ltimes \mathfrak{n})_{\mathrm{int}}^{*} / S \ltimes N$ of $(4.5)$ is open if and only if $C_{d}=C \cap \mathfrak{s}_{\mathrm{int}}^{*}$.

REMARK 4.11. In the present case the group $S \ltimes N$ is of type I, hence Priv $(S \ltimes N)$ can be identified with the unitary dual $(S \ltimes N)^{\wedge}$.

REMARK 4.12. When I was talking on this subject at the 1994 Oberwolfach conference on Harmonic Analysis I was kindly informed by E. O'Brien that precisely such a condition on cones as in 4.10 appeared in her study of a certain "saturation property" of (irreducible) finite-dimensional representations of complex semisimple Lie algebras. In her corresponding article, [16], such cones were called "normal". Interesting enough, this saturation property is closely related to the computation of $K$-groups of fixed point algebras under the action of compact groups on approximately finite $\left(C^{*}-\right)$ algebras, done by D. Handelman and $W$. Rossmann; for references see [16].

Proof. Suppose first that $\Omega$ is open, whence $\Lambda^{\prime}: \mathfrak{P} \rightarrow(\mathfrak{s} \ltimes \mathfrak{n})_{\text {int }}^{*} / S \ltimes N$ is open, and take a point in $C \cap \mathfrak{s}_{\text {int }}^{*}$, which may be written as $d \sigma=\sum_{j=1}^{n} r_{j} d \alpha_{j}$ with $\sigma \in S^{\wedge}$ and $r_{j} \in \mathrm{R}, r_{j} \geq 0$. Take any sequence $\left(\lambda_{k}\right)$ of positive real numbers converging to zero, and consider $\left(1, \lambda_{k}\right) \in S^{\wedge} \times \mathrm{R}^{\times} \subset \mathfrak{P}$ as well as $\left(\sigma^{-1}, 0\right) \in S^{\wedge} \times(N / Z N)^{\wedge} \subset \mathfrak{P}$ where $1 \in S^{\wedge}$ and $0 \in(N / Z N)^{\wedge}$ stand for the trivial characters. Using (1.18.iii) one easily sees that the sequence $\Lambda^{\prime}\left(1, \lambda_{k}\right)$ converges to the orbit $\Lambda^{\prime}\left(\sigma^{-1}, 0\right)$ : take $w_{k}^{(j)}=\left(2 r_{j} \lambda_{k}\right)^{\frac{1}{2}}$. Therefore, in view of Lemma 1.9 the sequence $\left(1, \lambda_{k}\right)$ converges to $\left(\sigma^{-1}, 0\right)$ in $\mathfrak{P}$, which means in particular that there exists a sequence $\left(m_{k}\right)$ in $\mathrm{N}_{0}^{n}$ with $\lim _{k \rightarrow \infty} \prod_{j=1}^{n} \alpha_{j}^{-m_{k}^{(j)}}=\sigma^{-1}$ or $d \sigma=\lim _{k \rightarrow \infty} \sum_{j=1}^{n} m_{k}^{(j)} d \alpha_{j}$. But the sequence $\sum_{j=1}^{n} m_{k}^{(j)} d \alpha_{j}$ in the lattice $\mathfrak{s}_{\text {int }}^{*}$ is constant eventually, whence $d \sigma \in C_{d}$.

For the reverse implication suppose $C_{d}=C \cap \mathfrak{s}_{\text {int }}^{*}$. In view of Theorem 3.39 and of $(1.15 / 18)$ it is enough to show if for a sequence $\left(\sigma_{k}, \lambda_{k}\right)$ in 
$S^{\wedge} \times \mathbf{R}^{\times} \subset \mathfrak{P}$ and a point $(\sigma, \gamma) \in S^{\wedge} \times(N / Z N)^{\wedge}$ the sequence $\Lambda^{\prime}\left(\sigma_{k}, \lambda_{k}\right)$ of orbits converges to $\Lambda^{\prime}(\sigma, \gamma)$ that then $\left(\sigma_{k}, \lambda_{k}\right)$ converges to $(\sigma, \gamma) \in \mathfrak{P}$. From Lemma 1.9 we conclude that we are free to pass to subsequences, in particular w.l.o.g. we may assume that all the $\lambda_{k}$ are positive. Proposition 1.18 provides us with a sequence $\left(w_{k}\right)$ in $\mathrm{C}^{n}$ such that $\lim _{k \rightarrow \infty} d \sigma_{k}-$ $\frac{1}{2 \lambda_{k}} \sum_{j=1}^{n}\left|w_{k}^{(j)}\right|^{2} d \alpha_{j}=d \sigma \quad$ and $\quad \lim _{k \rightarrow \infty} w_{k}^{(j)}=\gamma_{j} \quad$ for $\quad 1 \leq j \leq n \quad$ where $d \gamma(z)=\operatorname{Im} \sum_{j=1}^{n} \gamma_{j} \overline{z_{j}}$ for $z \in \mathrm{C}^{n}$.

Next choose a sequence $m_{k}^{\prime} \in \mathrm{N}_{0}^{n}$ such that $0 \leq \frac{1}{2 \lambda_{k}}\left|w_{k}^{(j)}\right|^{2}-m_{k}^{\prime(j)} \leq 1$ for all $k$ and all $j, 1 \leq j \leq n$. Passing to a subsequence we may assume that the sequences $\left(\frac{1}{2 \lambda_{k}}\left|w_{k}^{(j)}\right|^{2}-m_{k}^{\prime(j)}\right)$ converge for all $j$. Consider the sequence $\left(\varphi_{k}\right)$ in the lattice $\mathfrak{s}_{\text {int }}^{*}$ defined by

$$
\begin{aligned}
\varphi_{k} & =d \sigma_{k}-d \sigma-\sum_{j=1}^{n} m_{k}^{\prime(j)} d \alpha_{j}= \\
& =d \sigma_{k}-d \sigma-\frac{1}{2 \lambda_{k}} \sum_{j=1}^{n}\left|w_{k}^{(j)}\right|^{2} d \alpha_{j}+\sum_{j=1}^{n}\left(\frac{1}{2 \lambda_{k}}\left|w_{k}^{(j)}\right|^{2}-m_{k}^{\prime(j)}\right) d \alpha_{j} .
\end{aligned}
$$

From the second presentation it follows that $\left(\varphi_{k}\right)$ converges to a point $\varphi$ in $C$; as $\left(\varphi_{k}\right)$ is contained in $\mathfrak{s}_{\text {int }}^{*}$ it has to be constant eventually, hence $\varphi_{k}=\varphi \in C \cap \mathfrak{s}_{\text {int }}^{*}$ for $k \geq k_{0}$. By assumption there exist $m_{j}^{\prime \prime} \in \mathrm{N}_{0}$ such that $\varphi_{k}=\varphi=\sum_{j=1}^{n} m_{j}^{\prime \prime} d \alpha_{j}$. Defining $m_{k}^{(j)}=m_{k}^{\prime(j)}+m_{j}^{\prime \prime}$ provides us with a sequence needed to ensure that $\left(\sigma_{k}, \lambda_{k}\right)$ converges to $(\sigma, \gamma)$ in $\mathfrak{P}$. Note in passing that what we have proved above is essentially the following: If a collection of vectors $v_{1}, \ldots, v_{n}$ in a real vector space $V\left(=\mathfrak{s}^{*}\right)$ generates a lattice $\Gamma$, and if $C$ denotes the closed cone generated by $v_{1}, \ldots, v_{n}$ then the distance from $C$ to $\Gamma \backslash C$ is strictly positive.

Clearly, the above "discrete cone condition" is violated in many cases. The simplest example is obtained as follows. Since we also wish to discuss its simply connected version we write this down first.

Example 4.13. Let $\mathfrak{s}=S=\mathrm{R}$, and denote by $\eta: \mathrm{R} \rightarrow \mathrm{T}$ the function $\eta(s)=e^{i s}$. Take $n=2$, i.e., $N=N_{2}=\mathrm{C}^{2} \times \mathrm{R}$, and define $\alpha: S \rightarrow U$ by $\alpha_{1}=\zeta_{1} \circ \alpha=\eta^{3}$ and $\alpha_{2}=\zeta_{2} \circ \alpha=\eta^{2}$. The "lattice" $\Gamma$ generated by $d \alpha_{1}$ and $d \alpha_{2}$ is $\mathbf{Z} d \eta$, the cone $C$ is $\{r d \eta \mid r \geq 0\}$, hence $\Gamma \cap C=\left\{m d \eta \mid m \in \mathbf{N}_{0}\right\}$, while $C_{d}=\mathrm{N}_{0} d \alpha_{1}+\mathrm{N}_{0} d \alpha_{2}=(\Gamma \cap C) \backslash\{d \eta\}$. Of course, taking other relatively prime positive integers instead of 2 and 3 one can produce more and larger gaps. The group $S^{\prime}:=S / \operatorname{ker} \eta$ is a torus, the obvious action of $S^{\prime}$ on $N_{2}$ is faithful, and $\Gamma=\mathfrak{s}_{\text {int }}^{\prime *}$. Hence $S^{\prime} \ltimes N_{2}$ is a group as considered in Theorem 4.10 which does not satisfy the discrete cone condition.

We continue by discussing some particular sequences $\left(f_{k}^{\prime}\right)$ in $\left(\mathfrak{s} \ltimes \mathfrak{n}_{2}\right)^{*}$ of 
the above example. Choose any $\sigma \in S^{\wedge}$ (for instance an integral power of $\eta$ ) and any sequence $\left(\lambda_{k}\right)$ of positive real numbers converging to zero, and define $f_{k} \in\left(\mathfrak{s}^{*} \ltimes \mathfrak{n}_{2}\right)^{*}$ by $f_{k}=\Lambda\left(\sigma \eta, \lambda_{k}\right)$, i.e., by $f_{k}(A, z, t)=d \sigma(A)+d \eta(A)+$ $\lambda_{k} t$. Next choose a sequence $w_{k}=\left(w_{k}^{(1)}, w_{k}^{(2)}\right) \in \mathrm{C}^{2}$ such that

$$
\frac{1}{2 \lambda_{k}}\left(3\left|w_{k}^{(1)}\right|^{2}+2\left|w_{k}^{(2)}\right|^{2}\right)=1 \text {, }
$$

and both $w_{k}^{(1)} \lambda_{k}^{-\frac{1}{2}}$ and $w_{k}^{(2)} \lambda_{k}^{-\frac{1}{2}}$ converge to non-zero complex numbers $w^{(1)}$ and $w^{(2)}$.

By means of $\left(\frac{1}{\lambda_{k}} w_{k}, 0\right) \in N_{2}$ we transform $f_{k}$ to obtain $f_{k}^{\prime}:=\operatorname{Ad}^{*}\left(\frac{1}{\lambda_{k}} w_{k}, 0\right) f_{k}$, explicitly

$$
f_{k}^{\prime}(A, z, t)=d \sigma(A)+\lambda_{k} t+\operatorname{Im}\left(w_{k} \bar{z}\right) .
$$

Clearly, $\left(f_{k}^{\prime}\right)$ converges to $d \sigma$ because $\left(w_{k}\right)$ converges to zero. But the sequence of representations $\left(\pi_{k}\right)$ corresponding to $f_{k}^{\prime}$ (or to $f_{k}$ ) does not converge to $\sigma$ because the sequence $\left(\sigma \eta, \lambda_{k}\right)$ in $S^{\wedge} \times \mathbf{R}^{\times} \subset \mathfrak{P}$ does not converge to $(\sigma, 0) \in S^{\wedge} \times\left(N_{2} / Z N_{2}\right)^{\wedge}$. The latter claim is easily verified, clearly the reason is that $d \eta$ is missing in the "discrete cone" $C_{d}$, compare also the proof of (4.10).

Let's study the convergence in Fell's sense of the stabilizer groups $H_{f_{k}^{\prime}}$ of $f_{k}^{\prime}$ in $H:=S \ltimes N_{2}$ and the unitary characters $\vartheta_{k}^{\prime}$ on them corresponding to $f_{k}^{\prime}$. As $H_{f_{k}^{\prime}}=\left(w_{k} \lambda_{k}^{-1}, 0\right) H_{f_{k}}\left(-w_{k} \lambda_{k}^{-1}, 0\right)$ and $H_{f_{k}}=S \times Z N_{2}$ each $\left(s_{k}, z_{k}, t_{k}\right) \in H_{f_{k}^{\prime}}$ may be written as

$$
\begin{aligned}
& \left(s_{k}, z_{k}, t_{k}\right)=\left(w_{k} \lambda_{k}^{-1}, 0\right)\left(s_{k}, 0, r_{k}\right)\left(-w_{k} \lambda_{k}^{-1}, 0\right)=\left(s_{k}, w_{k}^{(1)} \lambda_{k}^{-1}\left(\alpha_{1}\left(s_{k}\right)^{-1}-1\right),\right. \\
& \left.w_{k}^{(2)} \lambda_{k}^{-1}\left(\alpha_{2}\left(s_{k}\right)^{-1}-1\right), r_{k}+\frac{1}{2}\left|w_{k}^{(1)}\right|^{2} \lambda_{k}^{-2} \operatorname{Im} \alpha_{1}\left(s_{k}\right)^{-1}+\frac{1}{2}\left|w_{k}^{(2)}\right|^{2} \lambda_{k}^{-2} \operatorname{Im} \alpha_{2}\left(s_{k}\right)^{-1}\right),
\end{aligned}
$$

and $\vartheta_{k}^{\prime}$ is given by

$$
\vartheta_{k}^{\prime}\left(s_{k}, z_{k}, t_{k}\right)=\vartheta_{k}\left(s_{k}, 0, r_{k}\right)=\sigma\left(s_{k}\right) \eta\left(s_{k}\right) e^{i \lambda_{k} r_{k}} .
$$

In other words, using $\alpha_{1}(s)=e^{3 i s}$ and $\alpha_{2}(s)=e^{2 i s}$, an element $y_{k}$ in $H_{f_{k}^{\prime}}$ looks like

$$
y_{k}=\left(s_{k}, w_{k}^{(1)} \lambda_{k}^{-1}\left(e^{-3 i s_{k}}-1\right), w_{k}^{(2)} \lambda_{k}^{-1}\left(e^{-2 i s_{k}}-1\right), t_{k}\right)
$$

with $s_{k}, t_{k} \in \mathrm{R}$, and $\vartheta_{k}^{\prime}\left(y_{k}\right)$ is given by

$$
\begin{aligned}
\vartheta_{k}^{\prime}\left(y_{k}\right) & =\sigma\left(s_{k}\right) \eta\left(s_{k}\right) e^{i \lambda_{k} t_{k}} e^{-\frac{1}{2} i \lambda_{k}^{-1}\left(\left|w_{k}^{(1)}\right|^{2} \sin \left(-3 s_{k}\right)+\left|w_{k}^{(2)}\right|^{2} \sin \left(-2 s_{k}\right)\right)} \\
& =\sigma\left(s_{k}\right) e^{i s_{k}+i \lambda_{k} t_{k}} e^{\frac{1}{2} \lambda_{k}^{-1}\left(\left|w_{k}^{(1)}\right|^{2} \sin 3 s_{k}+\left|w_{k}^{(2)}\right|^{2} \sin 2 s_{k}\right)}
\end{aligned}
$$

as $\eta(s)=e^{i s}$. 
Therefore, for a sequence $\left(y_{k}\right)$ in $H$ with $y_{k} \in H_{f_{k}^{\prime}}$ to converge (strictly speaking we would have to consider subsequences as well, but this would give the same result) it is necessary and sufficient that the four sequences $s_{k}, t_{k}, w_{k}^{(1)} \lambda_{k}^{-\frac{1}{2}}\left(e^{-3 i s_{k}}-1\right) \lambda_{k}^{-\frac{1}{2}}$, and $w_{k}^{(2)} \lambda_{k}^{-\frac{1}{2}}\left(e^{-2 i s_{k}}-1\right) \lambda_{k}^{-\frac{1}{2}}$ converge. Since by assumption $\left(w_{k}^{(j)} \lambda_{k}^{-\frac{1}{2}}\right)$ converges to $w^{(j)} \neq 0$ this means that $\left(e^{-3 i s_{k}}-1\right) \lambda_{k}^{-\frac{1}{2}}$ and $\left(e^{-2 i s_{k}}-1\right) \lambda_{k}^{-\frac{1}{2}}$ have to converge which (together with the convergence of $\left(s_{k}\right)$ ) forces $s_{k}$ to being $s_{k}=2 \pi m+\varepsilon_{k}$ where $m \in Z$ and $\left(\varepsilon_{k} \lambda_{k}^{-\frac{1}{2}}\right)$ is a convergent sequence of real numbers with limit $\mu$, say. Under these circumstances the sequences $\left(e^{-3 i s_{k}}-1\right) \lambda_{k}^{-\frac{1}{2}}$ and $\left(e^{-2 i s_{k}}-1\right) \lambda_{k}^{-\frac{1}{2}}$ converge to $-3 i \mu$ and $-2 i \mu$, respectively. We conclude that the sequence $\left(H_{f_{k}^{\prime}}\right)$ in Fell's space $\Sigma(H)$ of closed subgroups converges to

$$
H^{\prime}:=\left\{\left(2 \pi m,-3 i w^{(1)} \mu,-2 i w^{(2)} \mu, t\right) \mid m \in \mathbf{Z}, \mu, t \in \mathbf{R}\right\} .
$$

Next we consider the behaviour of $\vartheta_{k}^{\prime}\left(y_{k}\right)$ for convergent sequences $\left(y_{k}\right)$ in $H$ with $y_{k} \in H_{f_{k}^{\prime}}, y_{k}=\left(2 \pi m+\varepsilon_{k}, w_{k}^{(1)} \lambda_{k}^{-1}\left(e^{-3 i \varepsilon_{k}}-1\right), w_{k}^{(2)} \lambda_{k}^{-1}\left(e^{-2 i \varepsilon_{k}}-1\right), t_{k}\right)$ with limit $\left(2 \pi m,-3 i w^{(1)} \mu,-2 i w^{(2)} \mu, t\right)$, i.e., $\lim _{k \rightarrow \infty} \varepsilon_{k} \lambda_{k}^{-\frac{1}{2}}=\mu$ and $\lim _{k \rightarrow \infty} t_{k}=t$. As $\lambda_{k}^{-1} \varepsilon_{k}^{2}\left|w_{k}^{(j)}\right|^{2}$ tends to zero the sequence $\vartheta_{k}^{\prime}\left(y_{k}\right)$ behaves precisely as the sequence

$$
\sigma\left(2 \pi m+\varepsilon_{k}\right) e^{i \varepsilon_{k}+i \lambda_{k} t_{k}} e^{\frac{1}{2} \lambda_{k}^{-1}\left(\left|w_{k}^{(1)}\right|^{2} 3 \varepsilon_{k}+\left|w_{k}^{(2)}\right|^{2} 2 \varepsilon_{k}\right)}
$$

does, but in view of (4.14) the latter expression is nothing but

$$
\sigma\left(2 \pi m+\varepsilon_{k}\right) e^{2 i \varepsilon_{k}+i \lambda_{k} t_{k}},
$$

which converges to $\sigma(2 \pi m)=\sigma\left(\lim _{k \rightarrow \infty} y_{k}\right)$.

Summing up the sequence $\left(f_{k}^{\prime}, \vartheta_{k}^{\prime}\right)$ of Pukanszky parameters converges in a very natural sense to $d \sigma$, while the corresponding sequence $\left(\pi_{k}\right)$ in $H^{\wedge}$ does not converge to $\sigma$.

In view of the methods developed in this article, i.e., essentially using Takai duality to transform a $C^{*}$-algebra into a better tractable one, I suggest that before treating the case of general solvable Lie groups one should first understand the topology of the dual spaces of the following class of $C^{*}$-algebras, of course, in terms of parameters which are as canonical as possible. Let $Q=S \ltimes M$ be a semidirect product of a compact abelian Lie group $S$, not necessarily connected, and a simply connected R-polycyclic (in the sense of [1], sometimes called completely solvable) group $M$, and suppose that the commutator subgroup $[Q, Q]$ is a Heisenberg group. Moreover, let be given a homomorphism $\eta$ from a discrete finitely generated (free) abelian group $B$ 
into the Pontrjagin dual $(Q /[Q, Q])^{\wedge}$, which gives rise to an action $E^{\eta}$ of $B$ on $C^{*}(Q)$ in the sense of $(3.1)$, i.e.,

$$
\left(E_{b}^{\eta} \varphi\right)(y)=\varphi(x) \eta(b)(y)
$$

for $\varphi \in L^{1}(Q), \quad b \in B, \quad y \in Q$. Study these covariance algebras $C^{*}\left(B, C^{*}(Q), E^{\eta}\right)$. Special cases of such algebras were treated in the third section; but presumably not yet canonically enough.

\section{REFERENCES}

1. L. Auslander and B. Kostant, Polarization and unitary representations of solvable groups, Invent. Math. 14 (1971), 255-354.

2. P. Bernat, et alii, Représentations des groupes de Lie résolubles, Dunod, Paris 1972.

3. J. Boidol, et alii, Räume primitiver Ideale von Gruppenalgebren, Math. Ann. 236 (1978), 113.

4. I. Brown, Dual topology of a nilpotent Lie group, Ann. Sci. Éc. Norm. Sup. 6 (1973), 407411.

5. N. Conze and M. Duflo, Sur l'algèbre enveloppante d'une algèbre de Lie résolubles, Bull. Sci. Math. 94 (1970), 201-208.

6. J. Dixmier, Les $C^{*}$-algèbres et leurs représentations, Gauthier-Villars, Paris 1969.

7. J.M.G. Fell, A Hausdorff topology for the closed subsets of a locally compact non-Hausdorff space, Proc. Amer. Math. Soc. 13 (1962), 472-476.

8. J.M.G. Fell, Weak containment and induced representations of groups II, Trans. Amer. Math. Soc. 110 (1964), 424-447.

9. G.B. Folland, Harmonic Analysis in Phase Space, Ann. of Math. Stud. 122, 1989.

10. E.C. Gootman and J. Rosenberg, The structure of crossed product $C^{*}$-Algebras: A proof of the generalized Effros-Hahn conjecture, Invent. Math. 52 (1979), 283-298.

11. K.H. Hofmann, A memo on the exponential function and regular points, Arch. Math. 59 (1992), 24-37.

12. A.A. Kirillov, Unitary representations of nilpotent Lie groups, Uspeki Mat. Nauk 17 (1962), 53-104.

13. H. Leptin and J. Ludwig, Unitary Representation Theory of Exponential Lie Groups, Expositions in Math. 18, de Gruyter, Berlin 1994.

14. J. Ludwig, Dual topology of diamond groups, J. Reine Angew. Math. 467 (1995), 67-87.

15. O. Mathieu, Bicontinuity of the Dixmier map, J. Amer. Math. Soc. 4(1991), 837-863.

16. E. O'Brien, A saturation property for powers of irreducible characters, J. Reine Angew. Math. 456 (1994), 151-171.

17. D. Poguntke, Fell's subgroup algebra for locally compact abelian groups and $L^{1}$-covariance algebras, Colloq. Math. 69 (1995), 239-265.

18. L. Pukanszky, On the unitary representations of exponential groups, J. Funct. Anal. 2 (1968), $73-113$.

19. L. Pukanszky, The primitive ideal space of solvable Lie groups, Invent. Math. 22 (1973), 75118.

20. Takai, On a duality for crossed products of $C^{*}$-algebras, J. Funct. Anal. 19 (1975), 23-39.

21. E.T. Whittaker, and G.N. Watson, A Course of Modern Analysis, fourth edition, Cambridge Univ. Press 1963. 
22. D.P. Williams, The topology on the primitive ideal space of transformation group $C^{*}$-algebras and C.C.R. transformation group $C^{*}$-algebras, Trans. Amer. Math. Soc. 266 (1981), 335359 .

UNIVERSITÄT BIELEFELD

FAKULTÄT FÜR MATHEMATIK

POSTFACH 100131

33501 BIELEFELD

GERMANY 